JUDICIAL LAW-MAKING IN ENGLISH AND GERMAN COURTS 



\title{
JUDICIAL LAW-MAKING IN ENGLISH AND GERMAN COURTS
}

\author{
Techniques and Limits of Statutory \\ Interpretation
}

Martin BRENNCKE

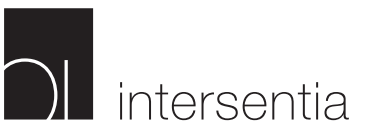

Cambridge - Antwerp - Chicago 
Intersentia Ltd

Sheraton House | Castle Park

Cambridge | CB3 0AX | United Kingdom

Tel.: +441223 370170 | Fax: +44 1223370169

Email: mail@intersentia.co.uk

www.intersentia.com | www.intersentia.co.uk

Distribution for the UK and Ireland:

NBN International

Airport Business Centre, 10 Thornbury Road

Plymouth, PL6 7PP

United Kingdom

Tel.: +44 1752202301 | Fax: +44 1752202331

Email: orders@nbninternational.com

Distribution for Europe and all other countries:

Intersentia Publishing nv

Groenstraat 31

2640 Mortsel

Belgium

Tel.: +32 36801550 | Fax: +3236587121

Email: mail@intersentia.be

Distribution for the USA and Canada:

Independent Publishers Group

Order Department

814 North Franklin Street

Chicago, IL 60610

USA

Tel.: +1 8008884741 (toll free) | Fax: +1 3123375985

Email: orders@ipgbook.com

Judicial Law-Making in English and German Courts. Techniques and Limits of Statutory Interpretation

(c) Martin Brenncke 2018

The author has asserted the right under the Copyright, Designs and Patents Act 1988, to be identified as author of this work.

No part of this book may be reproduced, stored in a retrieval system, or transmitted, in any form, or by any means, without prior written permission from Intersentia, or as expressly permitted by law or under the terms agreed with the appropriate reprographic rights organisation. Enquiries concerning reproduction which may not be covered by the above should be addressed to Intersentia at the address above.

Artwork on cover: (C) Praveetha Patalay, Flags

ISBN 978-1-78068-269-3

$\mathrm{D} / 2018 / 7849 / 94$

NUR 820

British Library Cataloguing in Publication Data. A catalogue record for this book is available from the British Library. 
For Praveetha 



\section{FOREWORD}

In Western democracies like Germany and the United Kingdom, the legal product of the democratic process is legislation. Legislation has to be read, understood and applied by a wide range of people. These include individual citizens, legal advisers, administrators and judges. When disputes arise, it is judges in courts who produce the authoritative final reading. But in doing that they have regard to how others are likely to have understood a statute. They also try to make sense of the legislation in the context of a range of other norms and values. Because legislation is everywhere and intrudes upon almost every legal relationship, statutory interpretation makes up a large part of what judges do and is one of the most important aspects of their work.

Working from the inside as a judge, the exercise of interpretation often feels more like an art than a science, even though one often feels a strong implicit sense of how to proceed. Yet the desirability of predictability in the application of law, which is inherent in the ideal of the rule of law, seems to demand that an objective science of interpretation be articulated and applied. Every legal system has to address the gap between the abstract statement of a law laid down in advance and its application to the facts of a particular case. There is no simple metric for weighing the relative normative force of different factors which may be relevant to giving determinate meaning to a statutory provision in its application to specific facts. Values of justice and reasonableness can operate as rather vague guides for how that gap is to be bridged. So can constitutional principles or a sense of hierarchy between norms bearing on the same subject matter as is addressed in the statute. Courts have to face up to the tension between positive statements of law in legislation and the pull of natural justice in spelling out the precise content of the legislative norm which they will identify as applicable to determine the dispute before them.

In the final analysis, it is the legal culture in a jurisdiction, generated within that jurisdiction's political and constitutional structures, which constrains judges in how far they feel able to go in imaginative interpretation of legislation. The ability of judges to give persuasive reasons for adopting one interpretation in preference to another is critical to the legitimacy of what they do. So some articulation of principle is required alongside the practice of interpretation, or as an inherent part of it. As Dr Brenncke explains, the use of determinate interpretative limits and techniques are important for reasons of legal certainty, upholding the proper separation of powers between judiciary and legislature 
and for the maintenance of public confidence in the judiciary. His book makes an important and sophisticated contribution to this endeavour, showing where lessons might be learned across the jurisdictional divide.

This book is a valuable study of how two jurisdictions approach the task of statutory interpretation in a complex and multivalent constitutional environment. It is the product of considerable scholarship across the two jurisdictions and a fine sensitivity to the various factors and different theoretical dimensions which inform the interpretative exercise. The exposition is clear. The argument is forceful. As with all the best works of comparative law, one reads this book and learns as much about one's own legal system as about the system with which it is compared.

As someone familiar with the English part of the comparison, I can attest that Dr Brenncke has a fine in-depth understanding of the processes of statutory interpretation used in the English courts. The detail of his exposition of the position in Germany shows that he has no lesser understanding of such processes in that jurisdiction. This makes him a guide to be listened to with attention.

Dr Brenncke's important theme is judicial law-making inherent in the process of statutory interpretation, and in particular the outer limits of what courts regard themselves as authorised to do in that regard. He rightly locates the practice of statutory interpretation in its constitutional setting in each jurisdiction. This important theoretical underpinning for what happens in judicial practice on the ground is sometimes obscured. The comparative approach reminds us of its importance and brings it to the fore. In Dr Brenncke's penetrating discussion, the practice of statutory interpretation becomes the basis for insights about the constitutional environment in both jurisdictions. Exploration of the outer boundaries of permissible judicial interpretations of legislation is a powerful way of tracing the practical implications of constitutional principles.

By comparing Germany and the United Kingdom (in particular, England \& Wales) using his detailed methodology, Dr Brenncke moves beyond the abstract platitudes one often finds in works which compare and emphasise the contrasts between civilian and common law jurisdictions. He reveals that there is a considerable degree of similarity in the approach of English and German judges to the practice of statutory interpretation.

That is especially so where important norms exterior to a statute have to be brought into account when reading the statute, in relation to the production of rights-consistent interpretations which take due account of specified human rights and interpretations which are required to conform, so far as possible, with EU law under the Marleasing ${ }^{1}$ principle. As Dr Brenncke points out, these interpretative obligations operate in tension with the desideratum of legal

Case C-106/89, Marleasing, ECLI:EU:C:1990:395. 
certainty in giving meaning to legislation; but if that value is given undue weight it would undermine the values which are served by those obligations and would reduce the effectiveness of human rights norms and of EU Directives. As he also observes, both approaches are likely to remain significant in English law after Brexit, notwithstanding the uncertainty as we write regarding the details of the post-Brexit settlement.

Rights-consistent statutory interpretation and interpretation in conformity with EU law are areas in which the constitutional settings in Germany and the United Kingdom have converged. The shift has been more marked for the United Kingdom, as a simple model of parliamentary sovereignty (with its own, relatively simple interpretative practice) has been replaced by a much more modulated model, which allows for the intrusion of different and competing constitutional values to a significant degree. Statutory interpretation in the United Kingdom has become more constitutionalised. The balance between will and reason, which is inherent in constitutional law and in the practice of statutory interpretation, has moved in the direction of reason. The simple statement of democratic will in the text of a statute has increasingly come to be read through the prism of constitutional reason. Nowadays, statutory meaning is derived less so by reference to simple textual analysis of what the legislating Parliament said, and increasingly by reference to an objective conception of meaning achieved through purposive interpretation and standards of reasonableness. Going still further beyond this, in relation to human rights and EU law the meaning of a statute is often the product of the judges' integration of the statutory text with human rights and EU norms. As Dr Brenncke says, the approach to interpretation becomes more result-driven (to identify what is the best rights- or EU law-compatible meaning which can be given to a statute) rather than processdriven (focusing on the process by which the statute came into existence). This is an intellectual process with which German courts have long been familiar through their own experience of the practice of statutory interpretation through integration with binding higher norms set out in Germany's constitutional Basic Law. It is fascinating to see how close interpretative practices have become in the two jurisdictions. Dr Brenncke's account bears out the impression I have obtained from discussion with German judges.

However, significant differences of approach remain, as Dr Brenncke is careful to acknowledge. Ordinary, conventional statutory interpretation is not so closely aligned in the two jurisdictions: see the discussion in Chapter 2. Again, I think he is right to trace the differences back to the respective constitutional contexts and the associated legal cultures in the two jurisdictions. The roots of the difference go back very far, reflecting the long parliamentary tradition in the United Kingdom. Franz Neumann, writing in the 1930s, observed that in the British doctrine of the Rule of Law associated with Dicey "the centre of gravity lies in the determination of the content of the laws by Parliament", while the German theory of the Rechtsstaat as developed in the 19th century 
"is uninterested in the genesis of the law, and is immediately concerned with the interpretation of a positive law, somehow and somewhere arisen"; "The German theory is liberal-constitutional; the English, democratic-constitutional." ${ }^{2}$ No doubt the German approach has now become more interested in the democratic genesis of the law, as Dr Brenncke explains. But British statutory interpretation remains primarily concerned with identifying the meaning of legislation as intended by the enacting Parliament, while the German conception of "objectivised intention" looks more to giving sense to legislation as read in the light of the current function it is taken to fulfil and of values at the time of its application in the present. The British doctrine of treating a statute as "always speaking" is not the same. As Dr Brenncke observes, this doctrine operates to help identify the intention of the legislating Parliament; it does not justify a court in departing from that intention.

This book reveals how German and British judges in many ways think in similar legal categories and engage in similar interpretative practices, participating in a real sense in a common European legal culture. It also shows how the openness and flexibility of the English common law and parliamentary tradition, with both its positive and its negative aspects, still remains distinct from the civilian tradition and the German approach to legal science and interpretation in important respects.

\author{
Philip Sales \\ Lord Justice of Appeal, \\ England \& Wales
}

2 F. Neumann, The Rule of Law: Political Theory and the Legal System in Modern Society, [1935-], Berg, Leamington Spa 1986, p. 185. 


\section{PREFACE}

Judicial Law-Making in English and German Courts addresses the often neglected relationship between statutory interpretation and constitutional law. The book is concerned with the limits of judicial power in a legal system. Judicial lawmaking occurs when judges restrict or extend the scope of application of a provision beyond or against the possible semantic meanings of the statutory language. How far do contemporary English and German judges go when they interpret national legislation? Where are the limits of statutory interpretation when judges venture outside the constraints of the text? Do these limits converge or diverge in both jurisdictions? The book critically analyses, reconstructs and compares judicial law-making in English and German courts from comparative, methodological and constitutional perspectives. It maps the differences and commonalities in both jurisdictions and then offers explanatory accounts for these differences and similarities based on constitutional, institutional, political, historical, cultural and international factors.

This book is addressed to a wide audience. It mainly appeals to an academic readership interested in the fields of statutory interpretation, legal methodology, constitutional law and comparative law. Practitioners in either jurisdiction will find it to be an accessible source of reference as this book reconstructs the fragmentary material on conventional, rights-consistent and EU-conforming judicial law-making in judicial opinions in English and German courts into a rational, coherent and systematic whole. Students of legal skills, constitutional law and comparative law will also find it a useful source of reference.

With regard to terminology, I use the term "case law" in a broad sense, meaning rulings of courts. The term "legislation" is solely used in this book to mean enacted law. "English courts" refers to the courts of England and Wales, and "English law" refers to the law applicable in England and Wales. The term "UK constitutional law" is used to refer to the constitutional law applicable in England. The term "provision" in this book refers to a specific section or part of a section either in primary or delegated legislation. "Construction" and "interpretation" are used interchangeably. All translations from German judgments are my own. Citations of German judgments do not link to the official reports as these are not available online, but rather to law journals where the judgments are published in full (these are available online via Beck-Online). Case law and literature are included up to 30 April 2018; Brexit-related legislation and legislative materials are included up to 1 August 2018. 
I am immensely indebted to Rolf Sethe, Jens Scherpe and Matthias Lehmann, who have provided support and guidance since the initial stages of my career and of this book. My particular thanks go to colleagues who have read and provided helpful comments on full chapters of this book, Frances Burton, Ryan Murphy and Gayatri Patel, and to colleagues who have provided helpful feedback on specific parts of this book, James Brown, Andrea Dolcetti, Binesh Hass, Pieter Koornhof, Kristie Thomas and Stephen Weatherill. I owe a special debt to Binesh Hass and Pradeep Patalay. Both of them shouldered the arduous task of checking the language in the manuscript. I am very grateful to the team at Intersentia, especially Ann-Christin Maak-Scherpe for her help, encouragement and lots of patience and Rebecca Moffat for an excellent job in seeing the book through production.

I want to thank the universities where I was based while working on this book: the University of Zurich, the University of Oxford and Aston University. I am also grateful to the universities and institutes where I held visiting positions during this period: the British Institute of International and Comparative Law, the University of Cambridge, the Institute of Advanced Legal Studies and the Max Planck Institute for Comparative and International Private Law. Special thanks are due to the Fritz Thyssen Stiftung für Wissenschaftsförderung and the Deutsch-Britische Juristenvereinigung for funding my visiting fellowship at the British Institute of International and Comparative Law.

Last but not least, I would like to acknowledge the immense patience and support shown by my family and friends while I worked on this book.

Martin Brenncke June 2018 


\section{CONTENTS}

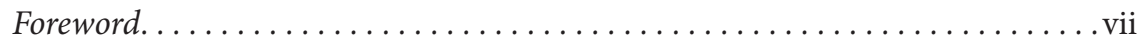

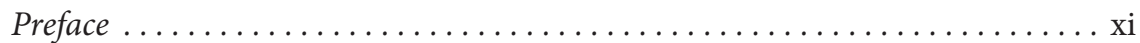

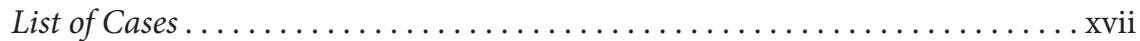

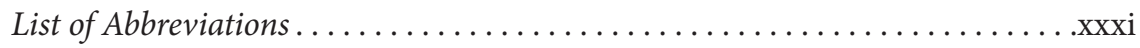

Chapter 1. Introduction $\ldots \ldots \ldots \ldots \ldots \ldots \ldots \ldots \ldots \ldots \ldots \ldots \ldots \ldots \ldots \ldots \ldots$

1. The Neglected Comparative Constitutional Law Perspective. ........... 3

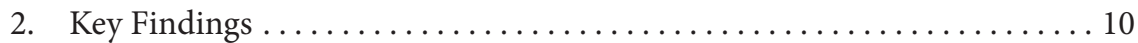

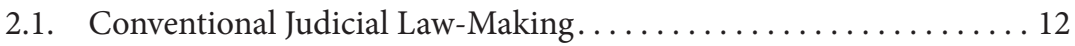

2.2. Rights-Consistent Judicial Law-Making . . . . . . . . . . . . 15

2.3. The European Legal Duty of Conforming Interpretation. . . . . . . . 20

2.4. The New Legal Duty of Conforming Interpretation after Brexit . . . . 26

2.5. Trends and Convergence in Judicial Law-Making. . . . . . . . . . 26

3. (Ir)relevance of Limits and Techniques of Interpretation . . . . . . . . 28

4. Method and Scope of the Book....................... 41

Chapter 2. Conventional Canons of Statutory Interpretation . . . . . . . . 49

1. Aim of Statutory Interpretation $\ldots \ldots \ldots \ldots \ldots \ldots \ldots \ldots \ldots$

2. The Interpretative Criteria and Their Weighing. ............. 54

2.1. Interpretation in a Narrow Sense $($ Auslegung $) \ldots \ldots \ldots \ldots \ldots \ldots 4$

2.2. Canons of Interpretation in England. . . . . . . . . . . . . . 58

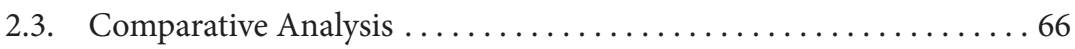

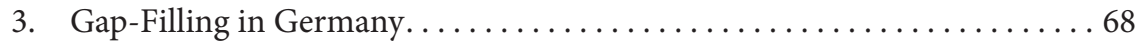

3.1. Judicial Law-Making Praeter Legem ................. 72

3.2. Judicial Law-Making Extra Legem ..................... 78

3.3. Constitutionalising the Limits of Statutory Interpretation. . . . . . 86

3.3.1. Common Limits, Different Application............ 87

3.3.2. The Recognised Methods of Statutory Interpretation ...... . 91

3.3.3. The Standard of Constitutional Review: Reasonableness . . . 94

3.3.4. Towards a Stricter Understanding of the Constitutional Limits of Interpretation $\ldots \ldots \ldots \ldots \ldots \ldots \ldots$

4. Reading In, Out or Down of Words in Legislation in England . . . . . . . 102

4.1. Literal Rule and the Doctrine of Parliamentary Sovereignty. . . . . 103

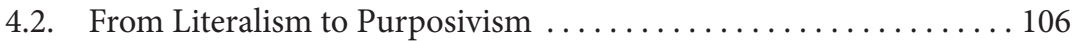


4.3. Saving Statutes from Absurdity ..................... . 114

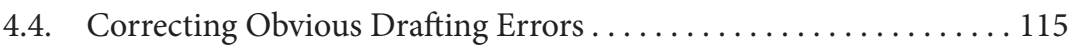

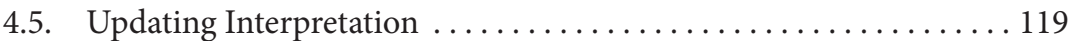

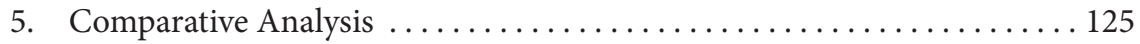

Chapter 3. Rights-Consistent Interpretation . . . . . . . . . . . . . . 139

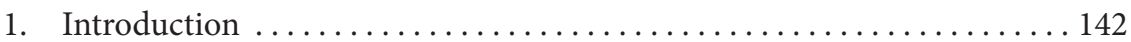

1.1. Constitution-Consistent Interpretation $\ldots \ldots \ldots \ldots \ldots \ldots \ldots \ldots \ldots \ldots \ldots$

1.2. Section 3(1) Human Rights Act . . . . . . . . . . . . . . . . . . . . . . . . 144

2. Aim of Rights-Consistent Interpretation. . . . . . . . . . . . . . 151

3. Relationship with Conventional Canons of Interpretation. . . . . . . . . . . 154

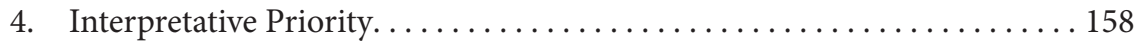

5. Presumption of Compliance . . . . . . . . . . . . . . . . . . . . 159

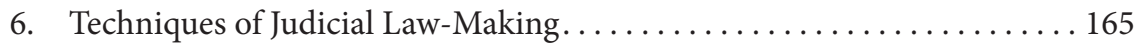

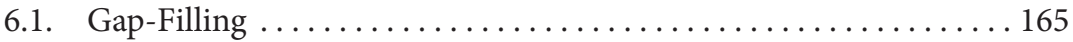

6.2. Reading In, Out or Down of Words in Legislation . . . . . . . . . 166

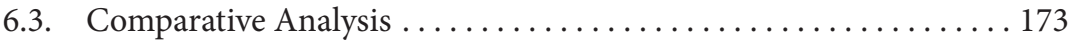

7. Outer Interpretative Limits . . . . . . . . . . . . . . . . . . . 179

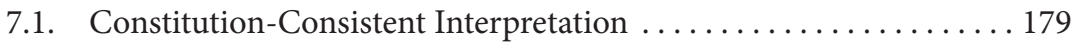

7.2. Section 3(1) Human Rights Act .................... 188

7.2.1. Statutory Language . . . . . . . . . . . . . . . 189

7.2.2. Intention of Parliament and Purpose of the Legislation . . . 197

7.2.3. Fundamental Features of the Legislation............ 208

7.2.4. Scheme, Grain and Thrust of the Legislation ........... 213

7.2.5. Policy Choices and Issues Calling for Legislative

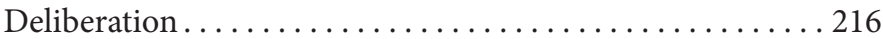

7.3. Comparative Analysis ... . . . . . . . . . . . . . . . . . . 223

7.3.1. Wide Powers of Rights-Consistent Judicial Law-Making . . . 225

7.3.2. High Level of Congruence.................. 228

7.3.3. The Constitutional Law Perspective............. 234

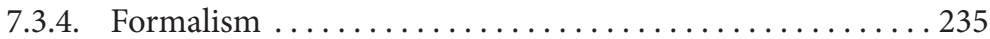

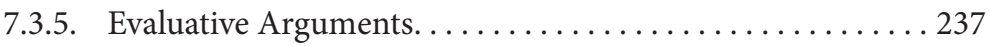

7.3.6. Legal (Un) certainty...................... 242

7.3.7. Convergence of Judicial Reasoning ............. 245

8. A Look into the Future: A UK Bill of Rights? ................. 246

Chapter 4. The European Legal Duty of Conforming Interpretation . . . . . 257

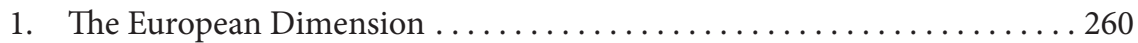

1.1. Scope of the EU Legal Duty of Conforming Interpretation. . . . . . 261

1.2. Functions of Conforming Interpretation . . . . . . . . . . 265

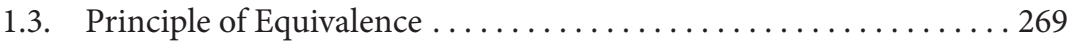


1.4. European Methodological Rules .................... 270

1.4.1. Interpretative Priority of the Conforming Meaning . . . . 272

1.4.2. Presumption of Compliance ................. 272

1.4.3. Relationship with National Legal Methodologies......... 275

1.5. The Contra Legem Limit . . . . . . . . . . . . . . . . . . . . . . 277

2. Aim of Conforming Interpretation . . . . . . . . . . . . . 279

3. Relationship with Other National Canons of Interpretation . . . . . . . . 288

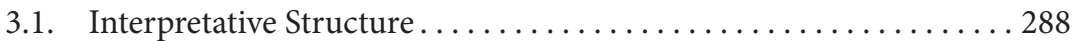

3.2. Conventional Limits of Judicial Law-Making . . . . . . . . . . . . 291

3.2.1. Rejection of a "Europeanisation from the Inside" in Germany . . . . . . . . . . . . . . . . . . . . 292

3.2.2. Endorsement of a "Europeanisation from the Inside"

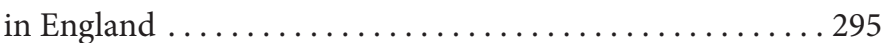

3.2.3. Comparative Analysis.......................... 309

3.3. Analogy to Rights-Consistent Interpretation. . . . . . . . . . . 311

3.3.1. Constitution-Consistent Interpretation in Germany....... 311

3.3.2. Section 3(1) Human Rights Act in England ........... 313

3.3.3. Comparative Analysis................................ 316

4. Interpretative Priority in National Courts. . . . . . . . . . . . . . . . . . . 318

5. Presumption of Compliance in National Courts . . . . . . . . . . . . . . . 319

5.1. The Presumption Rule in German Courts ............... . . . 319

5.2. The Presumption Rule in English Courts ............... 322

5.3. Comparative Analysis ... . . . . . . . . . . . . . . . . . . . . 331

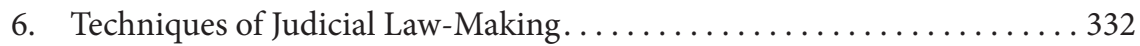

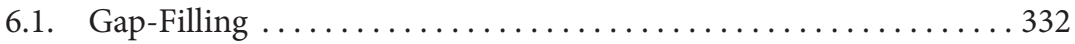

6.1.1. Legislation Adopted for the Purpose of Transposing

a Directive ............................. 332

6.1.2. Non-Implementing Legislation that Precedes a Directive . . . 338

6.2. Reading In, Out or Down of Words in Legislation . . . . . . . . . . . 343

6.3. Comparative Analysis . . . . . . . . . . . . . . . . . . . . 345

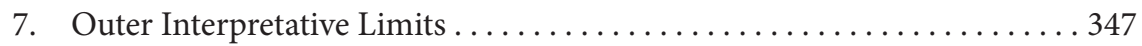

7.1. Limits of Conforming Judicial Law-Making in Germany ..... . . . 348

7.2. Limits of Conforming Judicial Law-Making in England . . . . . . . 358

7.2.1. Intention of the Enacting Parliament................... 359

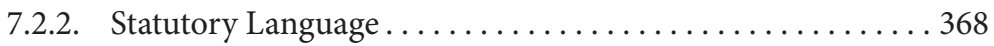

7.2.3. Scheme and Fundamental Features of the Legislation. ..... 372

7.2.4. Unsettled Outer Limits of Conforming Interpretation . . . . 374

7.3. Comparative Analysis . . . . . . . . . . . . . . . . . 380

7.3.1. Development Towards Converging Outer Limits of Conforming Judicial Law-Making . . . . . . . . . . 381

7.3.2. Evaluative Arguments and Judicial Discretion............ 385

7.3.3. Judicial Attitudes and the Stretching of the Outer Interpretative Limits 
7.3.4. Adaptability of the Outer Interpretative Limits 392

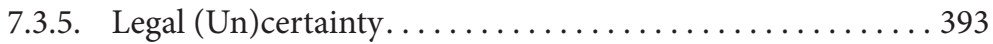

8. A Look into the Future: Brexit . . . . . . . . . . . . . . . . . . . . 396

8.1. Retained EU Law . . . . . . . . . . . . . . . . . . . . . . . . . . 398

8.2. The Principle of the Supremacy of EU Law .............. 401

8.3. The New Legal Duty of Conforming Interpretation . . . . . . . . . 406

8.4. Interpretation of Retained EU Law .................. 413

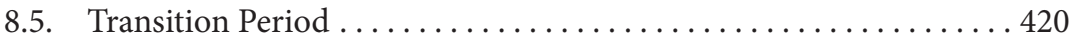

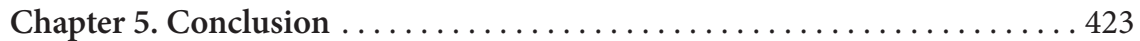

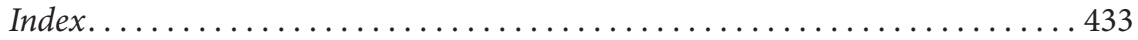

About the Author. ................................... 437 


\section{LIST OF CASES}

\section{COURT OF JUSTICE OF THE EUROPEAN UNION*}

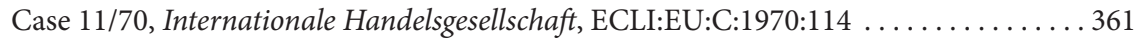

Case 43/75, Defrenne v. Sabena, ECLI:EU:C:1976:56 . . . . . . . . . . . . . . 305

Case 33/76, Rewe-Zentralfinanz eG and Rewe-Zentral AG

v. Landwirtschaftskammer für das Saarland, ECLI:EU:C:1976:188 . . . . . . . . 311

Case 106/77, Amministrazione delle Finanze dello Stato v. Simmenthal,

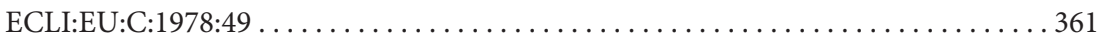

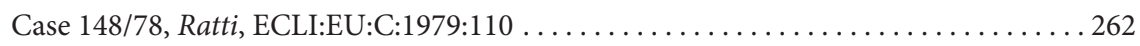

Case 61/81, Commission v. United Kingdom, ECLI:EU:C:1982:258 . . . . . . . . . 295

Case 14/83, von Colson and Kamann v. Land Nordrhein-Westfalen,

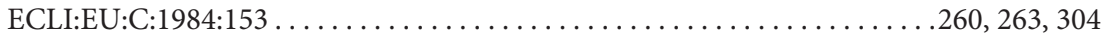

Case 79/83, Harz v. Deutsche Tradax, ECLI:EU:C:1984:155 . ............. 263, 304

Case 143/83, European Commission v. Denmark, ECLI:EU:C:1985:34 . . . . . . . . 395

Case 152/84, Marshall v. Southampton and South West Hampshire AHA,

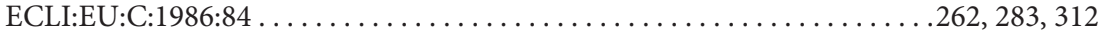

Case C-106/89, Marleasing v. La Comercial Internacional de Alimentación,

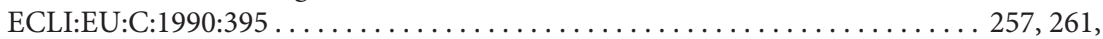

$277,285,303-304$

Case C-91/92, Faccini Dori v. Recreb Srl, ECLI:EU:C:1994:292 _. . . . . . .261-262, 313

Case C-334/92, Wagner Miret v. Fondo de Garantía Salarial,

ECLI:EU:C:1993:945 ....................... 261, 272-274, 330

Case C-421/92, Habermann-Beltermann v. Arbeiterwohlfahrt, ECLI:EU:C:1994:187 . . 263

Case C-31/93, Webb v. EMO Air Cargo (UK) Ltd, ECLI:EU:C:1994:300 . . . . . . . . . . 284

Case C-63/93, Duff v. Minister for Agriculture and Food and Attorney General,

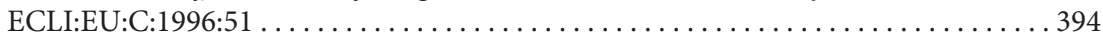

Case C-74/95, Criminal proceedings against X, ECLI:EU:C:1996:491_... . . . . 264, 396

Case C-168/95, Criminal proceedings against Arcaro, ECLI:EU:C:1996:363 . . . 262, 264, 396

Case C-129/96, Inter-Environnement Wallonie, ECLI:EU:C:1997:628 _. . . . . . . . . 265

Case C-197/96, Commission v. France, ECLI:EU:C:1997:155 . ................. 267

Case C-111/97, EvoBus Austria v. Niederösterreichische Verkehrsorganisations Gesellschaft mbH, ECLI:EU:C:1998:434 ....................... 260

Case C-78/98, Preston $v$. Wolverhampton Healthcare NHS Trust, ECLI:EU:C:2000:247 . . . . . . . . . . . . . . . . . . . . . . . . . . . . . . . 311

Case C-240/98, Océano Grupo Editorial v. Roció Murciano Quintero,

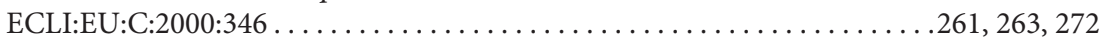

Case C-443/98, Unilever Italia v. Central Food, ECLI:EU:C:2000:496 . . . . . . . . . . . 262

Case C-456/98, Centrosteel Srl v. Adipol GmbH, ECLI:EU:C:2000:402 . . . . . . . . . 261

* Cases arranged chronologically. 


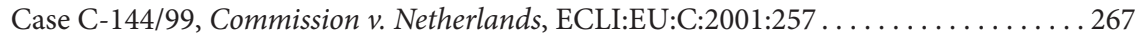

Case C-481/99, Heininger v. Bayerische Hypo- und Vereinsbank AG, ECLI:EU:C:2001:684 .................................294, 341

Case C-62/00, Marks \& Spencer plc. v. Commissioners of Customs and Excise,

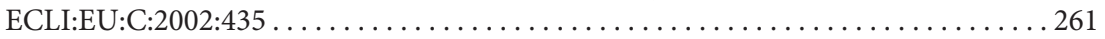

Case C-397/01, Pfeiffer v. Deutsches Rotes Kreuz, ECLI:EU:C:2004:584 . . . . 257, 261-262, $266,269,272-273,300,386,412$

Case C-371/02, Björnekulla Fruktindustrier v. Procordia Food,

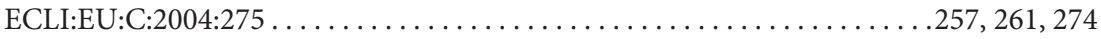

Case C-235/03, QDQ Media v. Alejandro Omedas Lecha, ECLI:EU:C:2005:147 . . . . . 277

Case C-144/04, Mangold v. Helm, ECLI:EU:C:2005:709. ..................262, 265

Case C-212/04, Adeneler et al. v. Ellinikos Organismos Galaktos, ECLI:EU:C:2006:443 ........................257, 261-262, 264-266, $272,276-277,300$

Case C-268/06, Impact v. Minister for Agriculture and Food and others,

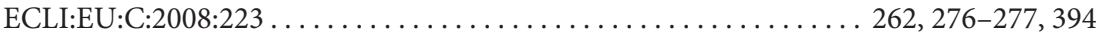

Case C-308/06, Intertanko v. Secretary of State for Transport, ECLI:EU:C:2008:312 ․ . 394 Case C-350/06, Schultz-Hoffv. Deutsche Rentenversicherung Bund, ECLI:EU:C:2009:18 . . . . . . . . . . . . . . . . . . . . . . . . . . . . . . . . . 341

Case C-404/06, Quelle v. Bundesverband der Verbraucherzentralen und

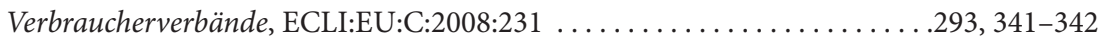

Case C-237/07, Janecek v. Freistaat Bayern, ECLI:EU:C:2008:447 . . . . . . . . 262, 268

Case C-378/07, Angelidaki and Others v. Organismos Nomarkhiaki Aftodiikisi Rethimnis, ECLI:EU:C:2009:250 . ............ 261, 264-265, 276-277

Case C-414/07, Magoora v. Dyrektor Izby Skarbowej w Krakowie,

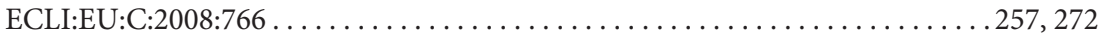

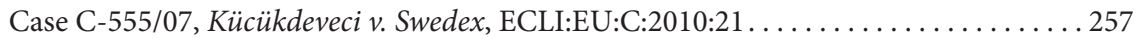

Case C-12/08, Mono Car Styling v. Dervis Odemis, ECLI:EU:C:2009:466 . . . . . 257, 262, $269,276-277,394$

Case C-63/08, Pontin v. T-Comalux, ECLI:EU:C:2009:666 ............... 311

Case C-201/08, Planatol v. Hauptzollamt Darmstadt, ECLI:EU:C:2009:539 _ . . . . . . 394

Case C-305/08, CoNISMa v. Regione Marche, ECLI:EU:2009:807 . . . . . . . . . . 257, 272

Cases C-403/08 and C-429/08, Football Association Premier League Ltd.

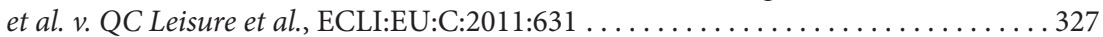

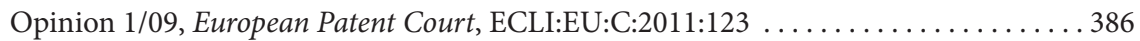

Case C-65/09, Gebr. Weber GmbH v. Wittmer, ECLI:EU:C:2011:396 _ . . . . . . . . . 334

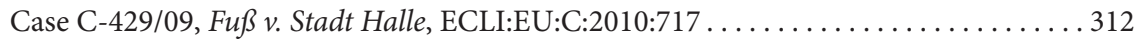

Case C-53/10, Land Hessen v. Franz Müksch OHG, ECLI:EU:C:2011:585 . . . . . . . . 264

Case C-214/10, KHS AG v. Schulte, ECLI:EU:C:2011:761 .................. 355

Case C-282/10, Dominguez v. Centre informatique du Centre Ouest Atlantique, ECLI:EU:C:2012:33 . ...................... 262-263, 266-267

Case C-621/10, Balkan and Sea Properties v. Direktor na Direktsia

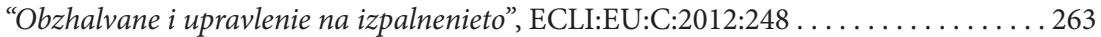

Case C-7/11, Criminal proceedings against Caronna, ECLI:EU:C:2012:396 _....... 257

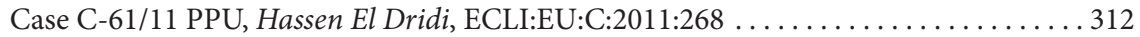

Case C-97/11, Amia v. Provincia Regionale di Palermo, ECLI:EU:C:2012:306 . . . 263, 266

Cases C-359/11 and C-400/11, Schulz and Egbringhoff, ECLI:EU:C:2014:2317 . . . . . 356

Case C-124/12, AES-3C Maritza East 1 EOOD v. Direktor na Direktsia "Obzhalvane i upravlenie na izpalnenieto" pri Tsentralno upravlenie na Natsionalnata agentsia za prihodite, Plovdiv, ECLI:EU:C:2013:488 ....................... 263 
Case C-142/12, Marinov v. Direktor na Direktsia "Obzhalvane i upravlenie

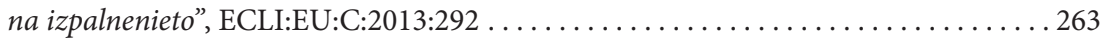

Case C-176/12, Association de médiation sociale v. Union locale des syndicats CGT,

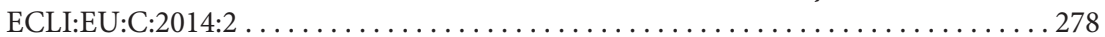

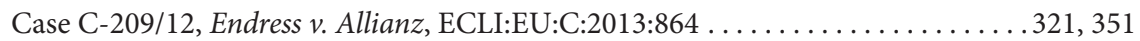

Case C-306/12, Spedition Welter v. Avanssur, ECLI:EU:C:2013:650 _. . 257, 263, 278, 393

Case C-539/12, Lock v. British Gas Trading Ltd., ECLI:EU:C:2014:351 . . . . . . . . 325

Case C-404/13, ClientEarth v. Secretary of State for the Environment,

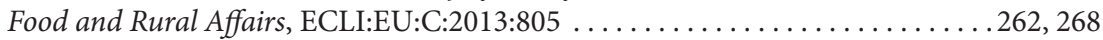

Case C-395/14, Vodafone GmbH v. Bundesrepublik Deutschland, ECLI:EU:C:2016:9 . . 337

Case C-441/14, Dansk Industri (DI) v. Estate of Karsten Eigil Rasmussen,

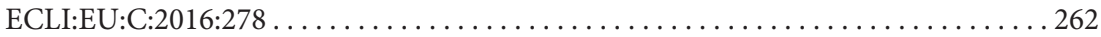

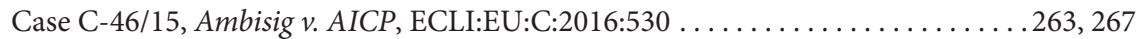

Case C-64/15, BP Europa v. Hauptzollamt Hamburg-Stadt,

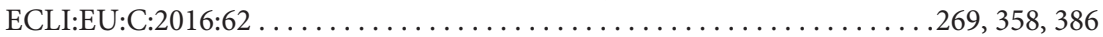

Case C-187/15, Pöpperl v. Land Nordrhein-Westfalen, ECLI:EU:C:2016:550 . . . . . . 263

Case C-231/15, Prezes Urzędu Komunikacji Elektronicznej and Petrotel v. Polkomtel,

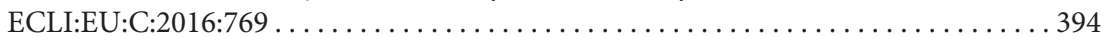

\section{GERMANY}

BAG, EuZW 2009, 465 (Schultz-Hoff) . . . . . . . . . . . . . 27. $289,311,319,340-341,348-349,387$

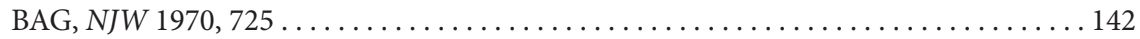

BAG, NJW 2003, 2473 ................................... 55, 72, 75

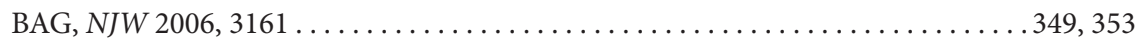

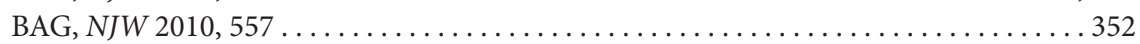

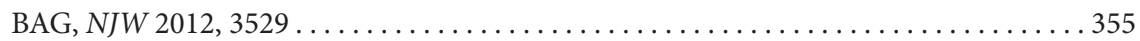

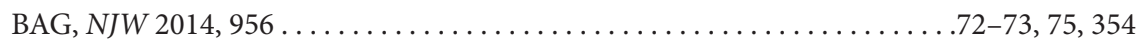

BAG, NZA 2003, $742 \ldots \ldots \ldots \ldots \ldots \ldots \ldots \ldots \ldots \ldots \ldots \ldots \ldots \ldots \ldots$

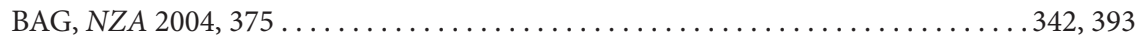

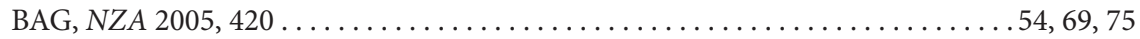

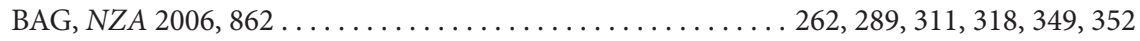

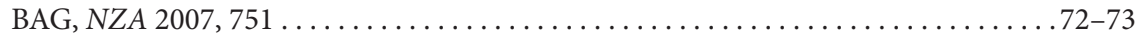

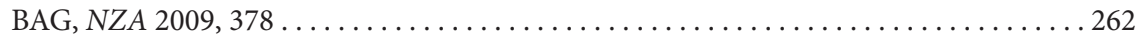

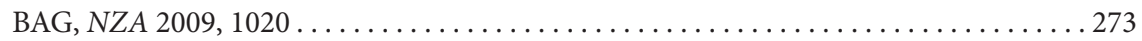

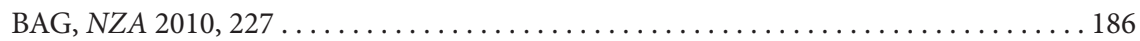

BAG, NZA 2010, 1020 (Urlaubsentgelt)................... 54-55, 174, 262,

$268,276,280,339-340,342$

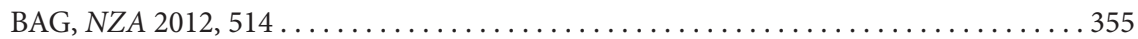

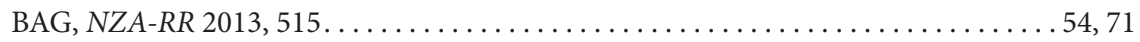

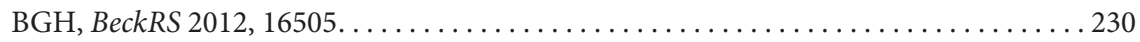

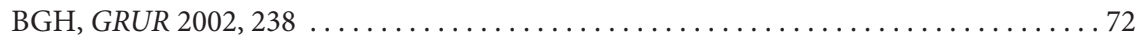

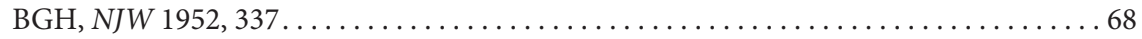

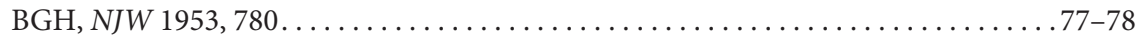

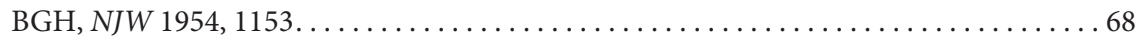

BGH, NJW 1955, 1276 (Tonband) .................. 58, 68, 73, 75, 92-93 
BGH, NJW 1955, 1433 (Fotokopie) $.58,73-74,92$

BGH, NJW 1958, 827 (Herrenreiter)

BGH, NJW 1963, 902 (Fernsehansagerin)

$.58,73,79-81$

BGH, NJW 1967, 343

$.49,51,56-58$

BGH, NJW 1969, 98.

222

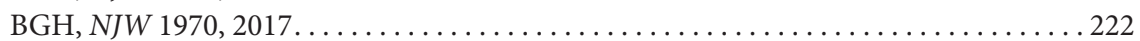

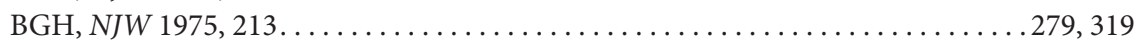

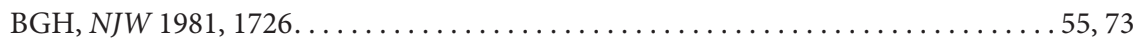

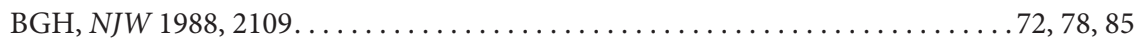

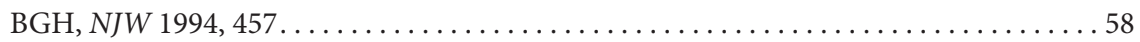

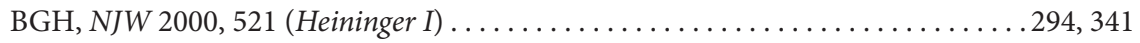

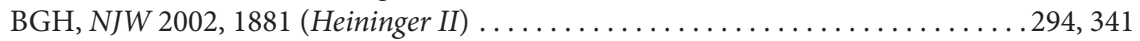

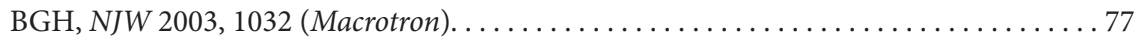

BGH, NJW 2006, 2997. . . . . . . . . . . . . . . . . . . . . . . . . . . 75

BGH, NJW 2006, 3200 (Quelle I) . . . . . . . . . . . . . . . . . . . . 292, 341, 352

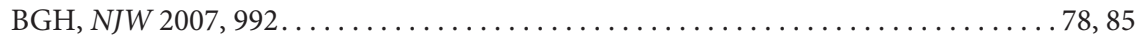

BGH, NJW 2007, 2419 (Rügeverkümmerung) . . . . . . . . . . . . . . . . . . 82

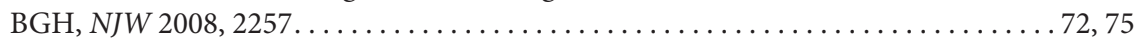

BGH, NJW 2008, 3213 (Dreiteilungsmethode) . . . . . . . . . . . . . . . . . 96

BGH, NJW 2009, 427 (Quelle II) . . . . . . . . . . . . . . . 54, 57, 71, 261,

$279,292-293,310,319,332$,

$334,342,347,348-350,353,393$

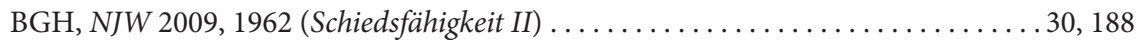

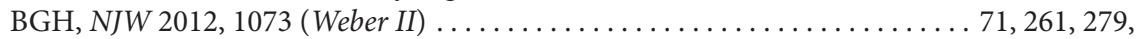

$292,310,319,321$,

$334-335,349-350,393$

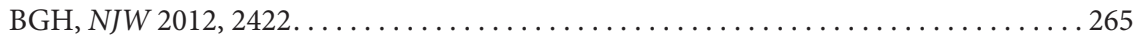

BGH, NJW 2013, 2674 (Interprofessionelle Sozietät) . . . . . . . . . . 49, 51, 55-56,

$230,273,280,289$,

$292,338,342,352$

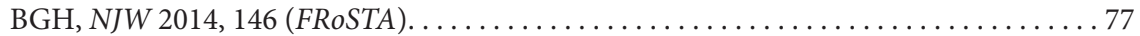

BGH, NJW 2014, 2646 (Lebensversicherung II) . . . . . . . . . . . . . . 999, 238, 272, 279,

290, 292, 321-322,

$327,335-336,349,353$

BGH, NJW 2015, 1023 (Lebensversicherung im Antragsmodell) . . . . . . . . . . . . . 351

BGH, NJW 2015, 3511 (Elektronische Leseplätze II) . . . . . . . . . . . . . . . . . . 338

BGH, NJW 2016, 1718 (Gasversorgung II) . . . . . . . . . . . . 71, 99, 349, 356-357

BGH, NJW 2017, 1093. . . . . . . . . . . . . . . . . . . . 57, 319, 349-350

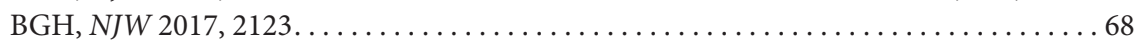

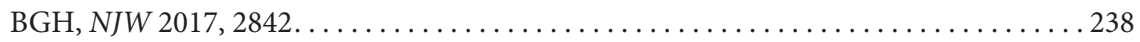

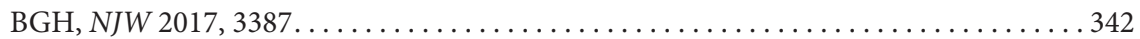

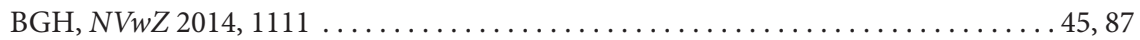

BGH, ZUM 2010, 429 (Bob Dylan (No. 2)). . . . . . . . . . . . . . . 292, 338, 349

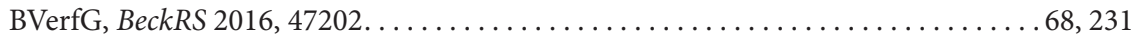

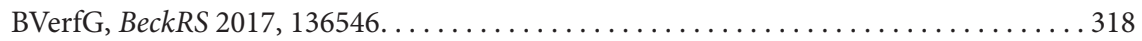

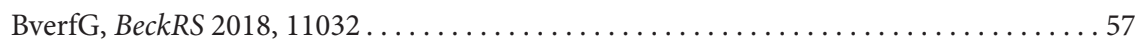

BVerfG, decision of 16.2 .2012 , no. 1 BvR $127 / 10 \ldots \ldots \ldots \ldots \ldots \ldots \ldots \ldots \ldots \ldots$

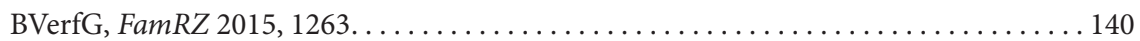

BVerfG, judgment of 31.20 .2016 , no. 1 BvR $871 / 13 \ldots \ldots \ldots \ldots \ldots \ldots \ldots \ldots \ldots \ldots \ldots$ 


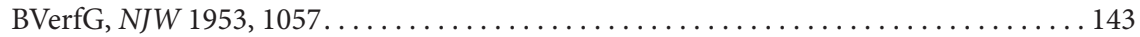

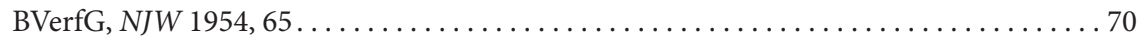

BVerfG, NJW 1958, 257 (Lüth) . . . . . . . . . . . . . . . . . . . . . . . 161

BVerfG, NJW 1958, 1227.............................. 87, 158, 180, 184

BVerfG, NJW 1958, 1388. . . . . . . . . . . . . . . . . . . . . . . . . . . . . . . 142

BVerfG, NJW 1958, 2059.................................... 186

BVerfG, NJW 1959, 1123.................................158, 179-180

BVerfG, NJW 1960, 1563.............................................. 49, 51-53, 56, 58

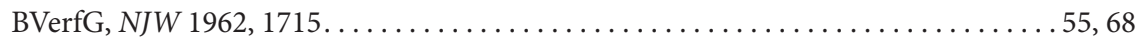

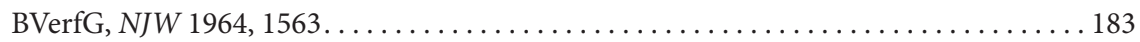

BVerfG, NJW 1965, 1427................................ 142, 158, 161

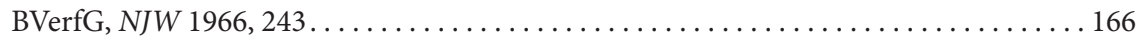

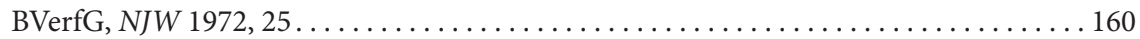

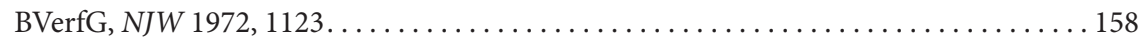

BVerfG, NJW 1972, 1934....................................... 179

BVerfG, NJW 1973, 1221 (Soraya) ........................ 51, 58, 69, 73, 78-79,

$81-82,84,86,88,222,238$

BVerfG, NJW 1973, 1491.............................. 55-56, 68,

$86-87,142,155,158,160$

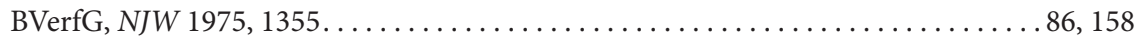

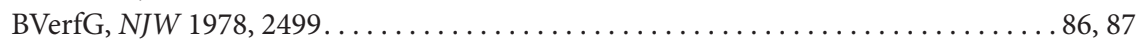

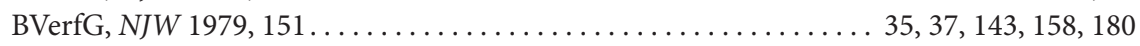

BVerfG, NJW 1979, 305 (Sachverständigenhaftung) ........... 36, 68, 87-88, 126

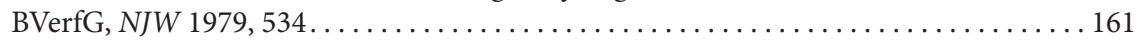

BVerfG, NJW 1979, 1925...................................... 37

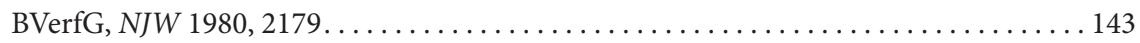

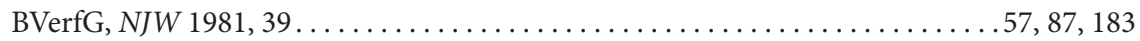

BVerfG, NJW 1982, 1375 . . . . . . . . . . . . . . . . . . . . . . . . 179-180

BVerfG, NJW 1982, 1509..................................... 180

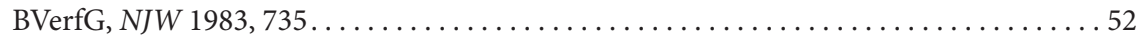

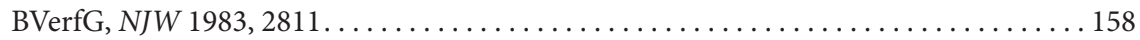

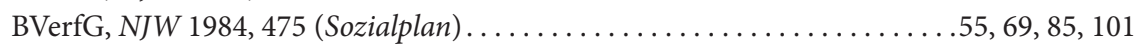

BVerfG, NJW 1985, 1519...................................... 143, 180

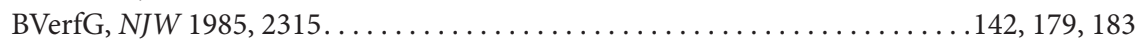

BVerfG, NJW 1985, 2395 ...................................... 86

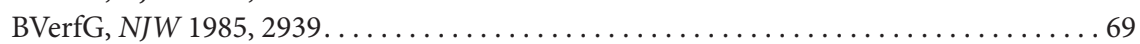

BVerfG, NJW 1986, 1672 ....................................... 87

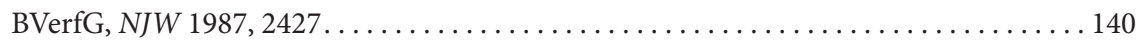

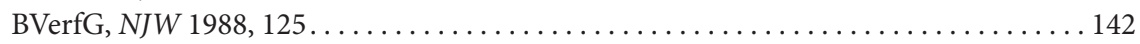

BVerfG, NJW 1988, 1902 ..................................... 87

BVerfG, NJW 1990, 1593............................ 31, 35, 58,

$69,73-75,81,87,90$,

$94-95,126,226,238$

BVerfG, NJW 1991, 1807. . . . . . . . . . . . . . . . . . . . . . 143

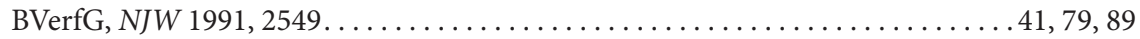

BVerfG, NJW 1992, 890 (Eilversammlungen) . ...................180-181, 184

BVerfG, NJW 1992, 1219. . . . . . . . . . . . . . . . . . . . . . . . . . 92

BVerfG, NJW 1992, 2947..................................... 143, 158

BVerfG, NJW 1993, 996................................... 79, 94

BVerfG, NJW 1993, 1379 (Streikeinsatz von Beamten) . . . . . . . . . . . . . . . . . 89 
BVerfG, NJW 1994, 2475. $87,143,183$

BVerfG, NJW 1995, 1141 . .

BVerfG, NJW 2009, 499 . . . . . . . . . . . . . . . . . . . . . . . . . . . . 98

BVerfG, NJW 2009, 1469 (Rügeverkümmerung)

$6,41,58,79,83$,

$86-88,90-91,94,96,100,136$

BVerfG, NJW 2011, 819. . .

BVerfG, NJW 2011, 842 (Dreiteilungsmethode)

$91,96-99,101,348$

BVerfG, NJW 2011, 1723 $.73,87,94,98$

BVerfG, NJW 2012, 669 . $35,39,45,94,294$,

$311,318-319,347-350,393$

BVerfG, NJW 2012, 2639 . . 88-89

BVerfG, NJW 2012, 3081 (Delisting) $49,55,57,73,77$,

$86-87,91,94-95,98-99,348$ BVerfG, NJW 2013, 1058 (Deal im Strafprozess) ........... 49, 51, 56, 58, 68, 87, 90 BVerfG, NJW 2014, 1874 (Flashmob) . . . . . . . . . . . . . . . . . . . 78

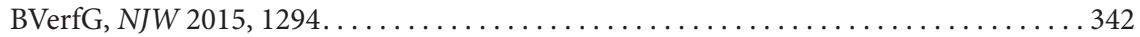
BVerfG, NJW 2015, 1359 (Kopftuch) ............... 58, 140, 155, 183, 185-186, 188 BVerfG, NJW 2015, 1506 (Scheinvater) . . . . . . . . . . . . . . . . . . 88-89 BVerfG, NJW 2015, 2549..................................... 44 BVerfG, NJW 2015, 2949. . . . . . . . . . . . . . . . . . . . . . .94, 158, 160 BVerfG, NJW-RR 2016, $1366 \ldots \ldots \ldots \ldots \ldots \ldots \ldots \ldots \ldots \ldots \ldots \ldots \ldots \ldots \ldots \ldots . \ldots \ldots, 49,86-87$, 


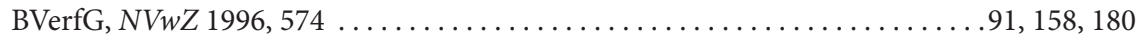

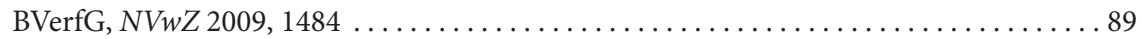

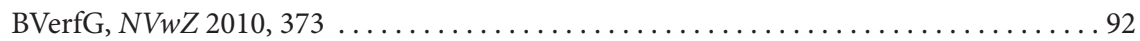

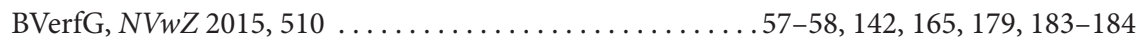

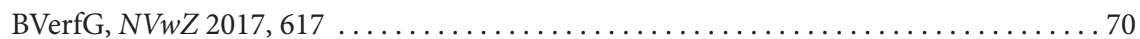

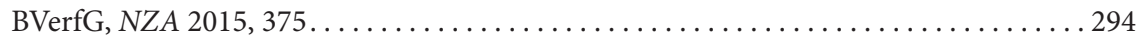

BVerfG, NZA 2015, 1117........................................ 79

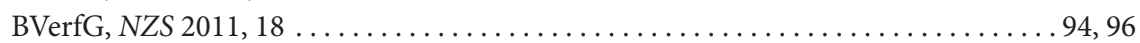

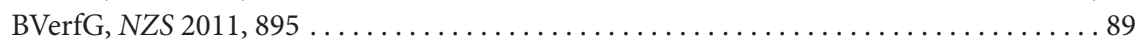

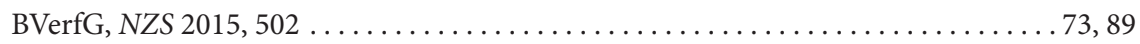

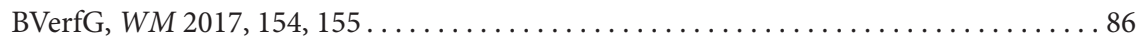

BVerwG, BeckRS 2017, 103948................... 45, 322, 337-338, 353

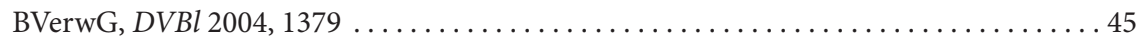

BVerwG, judgment of 29.1.2004, no. 3 C $39.03 \ldots \ldots \ldots \ldots \ldots \ldots \ldots \ldots 261,291,318$

BVerwG, judgment of 10.08 .2016 , no. 1 B 82/16 (1 PKH 76.16) .............. 87

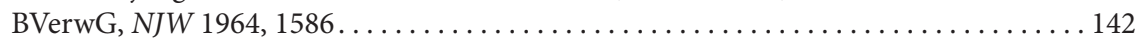

BVerwG, NJW 2005, 1293 ...................................... 73

BVerwG, $N V w Z$ 1997, 384................................... 142, 161

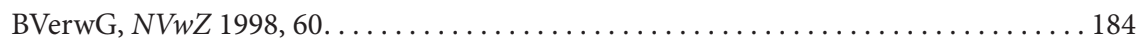

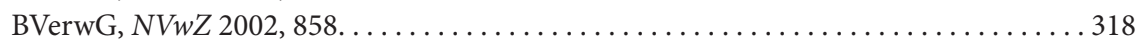

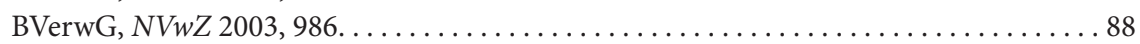

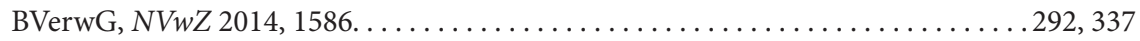

LG Hamburg, decision of 1.2.1988, no. 11 S 398/87 ...................... 74

\section{UNITED KINGDOM}

9 Cornwall Crescent London Ltd. v. Kensington and Chelsea RLBC

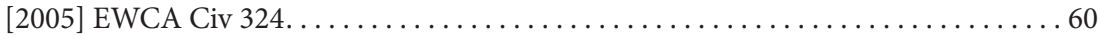

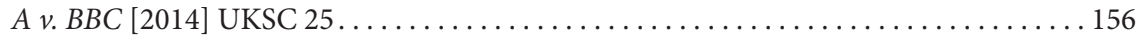

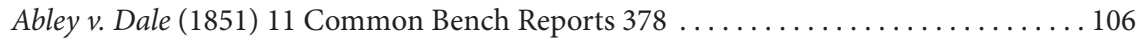

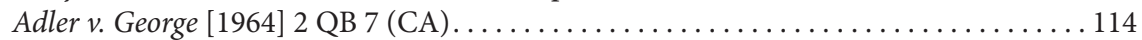

Advocate General for Scotland v. MacDonald [2003] UKHL 34 ................... 53

Ahmed and others $v$. HM Treasury [2010] UKSC $2 \ldots \ldots \ldots \ldots \ldots \ldots \ldots$. . . . . . .

$201,248,250$

Airtours Holidays Transport v. Revenue and Customs Commissioners

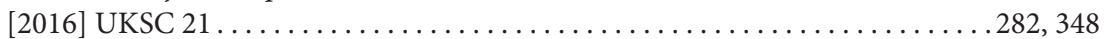

Alderson v. Secretary of State for Trade and Industry [2003] EWCA Civ 1767 . . . . 300, 318

Anisminic v. Foreign Compensation Commission [1969] 2 AC 147 (HL) .........251, 255

AS (Somalia) v. Secretary of State for the Home Department [2009] UKHL 32 . . . . 193, 196

Assange v. Swedish Prosecution Authority [2012] UKSC 22 ............... 65, 299,

$314,323,372,374$

Associated Newspapers Ltd. v. Wilson [1995] 2 AC 454 (HL)................62, 104

Attorney General's Reference (No. 4 of 2002) [2004] UKHL 43................ 144, 153,

$169,172,197-198$

Attorney-General v. Prince Ernest Augustus of Hanover [1957] AC 436 (HL) . .. .52, 63, 273

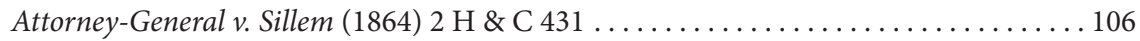


Austin v. Mayor and Burgesses of the London Borough of Southwark

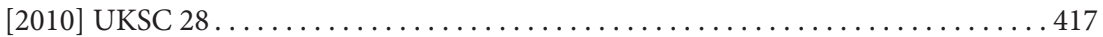

AXA General Insurance Ltd. v. Lord Advocate [2011] UKSC 46 . . . . . . 110, 125, 248, 256

Axa UK Plc. v. Revenue and Customs Commissioners [2011] EWCA Civ 1607 . . . . . 322

$B$ (a child) v. DPP [2000] 2 AC 428 (HL) ............................ 4

$B$ (Algeria) v. Secretary of State for the Home Department [2018] UKSC 5 . . . . . . . 251

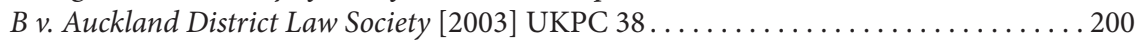

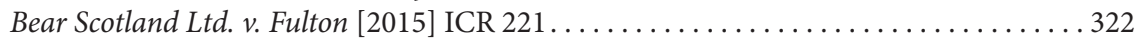

Bearmans Ltd. v. Metropolitan Police District Receiver [1961] 1 WLR 634 (CA)........ 60

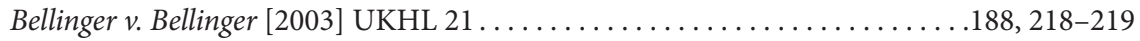

Benkharbouche v. Embassy of Sudan [2014] ICR $169 \ldots \ldots \ldots \ldots \ldots \ldots \ldots . \ldots . \ldots . \ldots . \ldots 23$

Benkharbouche v. Embassy of Sudan [2015] EWCA Civ $33 \ldots \ldots \ldots .145,210,223,373$

Birmingham City Council v. Doherty [2008] UKHL 57...................... 191

Birmingham Hippodrome Theatre Trust Ltd. v. Revenue and Customs

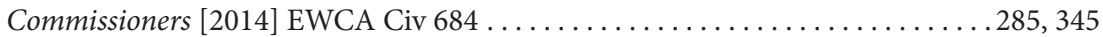

Black-Clawson International v. Papierwerke Waldhof-Aschaffenburg

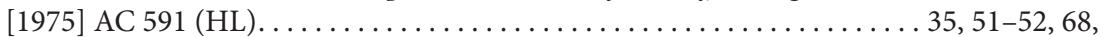

$99,103-104,132,134$

Blackwood v. Birmingham and Solihull Mental Health NHS

Foundation Trust [2016] EWCA Civ 607

$.45,290,359,373$

Bloomsbury International Ltd. v. Sea Fish Industry Authority [2011] UKSC 25 . . . 60, 62

Bogdanic v. The Secretary of State for the Home Department

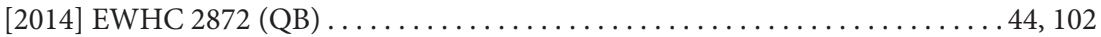

Brent LBC v. Risk Management Partners Ltd. [2011] UKSC 7 ................... 323

British Airways plc. v. Williams [2012] UKSC 43 .......................... 323

British Gas Trading Ltd. v. Lock [2016] EWCA Civ 983 ............... 258, 261, 274,

290, 314, 323, 325-326, 344,

$348,363-364,374-375$

Cachia v. Faluyi $[2001]$ EWCA Civ 998. ............................. 156

Carter v. Bradbeer [1975] 1 WLR 1204 (HL) ......................61, 63, 106

Cartier International AG v. British Sky Broadcasting Ltd. [2014] EWHC 3354 . . . . . . 281

Cartier International AG v. British Sky Broadcasting Ltd.

[2016] EWCA Civ 658 . . . . . . . . . . . . . . . . . . . . . . . . . . . . . . . . 290, 299

Cheng v. Governor of Pentonville Prison [1973] AC 931 (HL) . . . . . . . . . . . . .61-62

Churchill Insurance Co. Ltd. v. Fitzgerald \& Wilkinson

[2012] EWCA Civ 1166.

$45,189,201,215-216$

$285,299,314,323$,

$345,368,370,372-374$

Clark \& Tokeley Ltd. (t/a Spellbrook) v. Oakes [1999] ICR 276 (CA) . .............60 60

Clarke v. Kato [1998] 1 WLR 1647 (HL) ..........................102, 289, 308

Cooper v. HM Attorney General [2010] EWCA Civ 464 ...................... 347

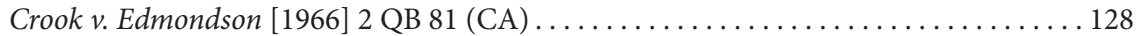

Cutler v. Wandsworth Stadium Ltd. [1949] AC 398 (HL) . . . . . . . . . . . . . . . . . . . . . 108

Dabas v. High Court of Justice in Madrid [2007] UKHL 6 . . . . . . . . . . . . . . . . 314

Day v. Lewisham and Greenwich NHS Trust [2017] EWCA Civ 329 .............. 113

Digital Satellite Warranty Cover Ltd. v. The Financial Services Authority

[2011] EWCA Civ 1413...................................45, 314

Dingmar v. Dingmar [2006] EWCA Civ $942 \ldots \ldots \ldots \ldots \ldots \ldots \ldots \ldots \ldots . \ldots \ldots, 108,114$

Director of the Serious Fraud Office v. B [2012] EWCA Crim 901 . . . . . . . . . . . . . . 118

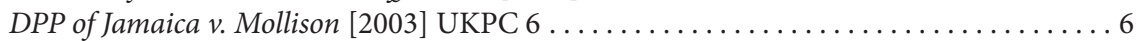


Duke v. GEC Reliance Ltd. [1988] AC 618 (HL)........... 52, 271, 307, 283, 347, 367 Duport Steels Ltd. v. Sirs [1980] 1 All ER 529 (HL) ......... . 6, 32, 51, 61, 104, 238-239 EB Central Services Ltd. v. Revenue and Customs Commissioners

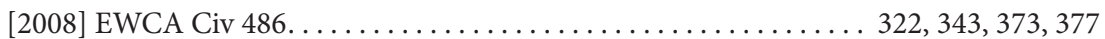

EBR Attridge Law LLP v. Coleman [2010] ICR 242 .................... 265, 343

Ellen Street Estates v. Minister of Health [1934] 1 KB 590 (CA). . . . . . . . . . . . . . . . 5, 204

EN (Serbia) v. Secretary of State for the Home Department

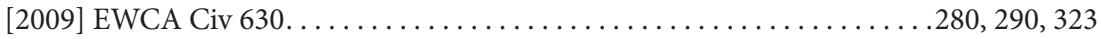

English v. Thomas Sanderson Blinds Ltd. [2008] EWCA Civ 1421................ 374

F Hoffmann-La Roche \& Co. v. Secretary of State for Trade and Industry

[1975] AC 295 (HL)............................................. 306

Finnegan v. Clowney Youth Training Programme Ltd. [1990] 2 AC 407 (HL) ......... 304

Fitzpatrick v. Sterling Housing Association Ltd. [2001] 1 AC 27 (HL) ........... 2-3, 52,

$120-122,124,126,167,190$

Fleming (t/a Bodycraft) v. Customs and Excise Commissioners

[2006] EWCA Civ 70........................ 34, 177, 343, 345, 369

Fleming (t/a Bodycraft) v. Customs and Excise Commissioners [2008] UKHL 2 . . . . . 378 Football Association Premier League Ltd. v. QC Leisure

[2012] EWCA Civ 1708....................................282, 327, 369

Football Association Premier League Ltd. v. QC Leisure

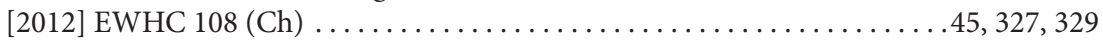

Fothergill v. Monarch Airlines Ltd. [1981] AC 251 (HL) ................... 108, 134

Garland v. British Rail Engineering Ltd. [1983] 2 AC 751 (HL) ..........65, 106, 281, 367

Ghaidan v. Godin-Mendoza [2004] UKHL 30 ..................3-4, 39, 144-147,

$152-153,155-156,159,162$,

$167-170,172,178,181,188-195$,

197-198, 200, 202-203, 205, 208,

210-211, 213-217, 220-221, 227,

$229,299,313,343$

Ghany v. Attorney General of Trinidad and Tobago [2015] UKPC 12 . . . . . . . . . 117-118

Gladstone v. Bower [1960] 2 QB 384 (CA) .........................102-103, 134

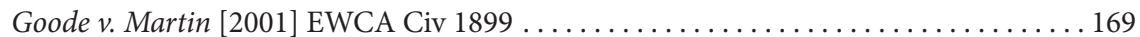

Google Inc. v. Vidal-Hall [2015] EWCA Civ $311 \ldots \ldots \ldots \ldots \ldots \ldots \ldots 4,314,343$,

$347,372,375-377$

Greenweb Ltd. v. London Borough of Wandsworth [2008] EWCA Civ 910. . . .113, 118, 128 H. v. Lord Advocate [2012] UKSC 24 ........................... 110, 203

Hamnett v. Essex County Council [2017] EWCA Civ 6...................... 5

Hampshire v. Board of the Pension Protection Fund [2016] EWCA Civ 786. . . . . . 45, 372

Hemming ( $t$ /a Simply Pleasure Ltd) v. Westminster City Council

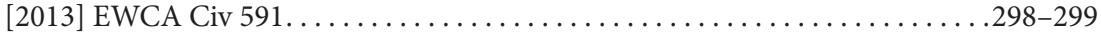

Hill v. East and West India Dock Co. (1884) 9 AC 448 (HL) .................... 106

Holden \& Co v. Crown Prosecution Service (No. 2) [1994] 1 AC 22 (HL).......... 112, 128

Holmes v. Bradfield Rural District Council [1949] 2 KB 1..................... 61

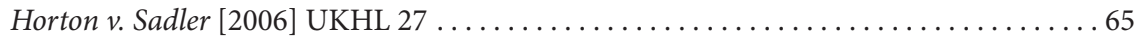

Hounslow London Borough Council v. Powell [2011] UKSC $8 \ldots \ldots \ldots \ldots \ldots \ldots \ldots . \ldots 210$

Howe v. Motor Insurers' Bureau [2017] EWCA Civ $932 \ldots \ldots \ldots \ldots \ldots . .258,299,374$

In re S (Minors) [2001] EWCA Civ 757 . . . . . . . . . . . . . . . . . . . . . . . . . . . 209

In re S (Minors) [2002] UKHL 10 . . . . . . . . . . . . . . . . . . . 145, 147, 156,

$169,188,194,208-209,218$

Inco Europe Ltd. v. First Choice Distribution [2000] 1 WLR 586 (HL) . . 102, 115-116, 118 
Inland Revenue Commissioners v. Hinchy [1960] AC 748 (HL) .................. 53 Inland Revenue Commissioners v. McGuckian [1997] 1 WLR 991 (HL) . . . . . . . . . 106 ITV Broadcasting Ltd. v. TV Catchup Ltd. (No. 2) [2011] EWHC 1874 . . . . . . . . . 260, 299,

ITV Broadcasting Ltd. v. TV Catchup Ltd. [2015] EWCA Civ $204 \ldots \ldots \ldots \ldots . .45,324$, $343,347,372,374,384$ Johnson v. Medical Defence Union Ltd. [2007] EWCA Civ 262 .................. 383

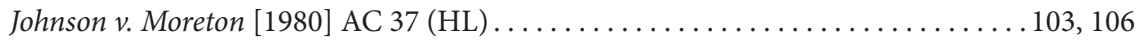

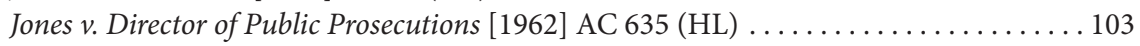
Jones $v$. Secretary of State for Social Services [1972] AC 944 (HL) ..................6 61 Jones v. Wrotham Park Settled Estates [1980] AC 74 (HL) ........... 103, 108, 116-117, $119,123,129$

Kammins Ballrooms Co Ltd. v. Zenith Investments (Torquay) Ltd.

[1971] AC 850 (HL) . . . . . . . . . . . . . . . . . . . . . . . . . . . . . 103, 117 Kay Green v. Twinsectra Ltd. [1996] 1 WLR 1587 (CA) . . . . . . . . . . . . . . . . 113 Kennedy v. The Charity Commission [2014] UKSC $20 \ldots \ldots \ldots \ldots \ldots$. ....... 210, 215, 249 Kirkness (Inspector of Taxes) v. John Hudson \& Co. Ltd. [1955] AC 696 (HL) . . . . . 63, 273 Lehman Brothers International (Europe) (In Administration), Re

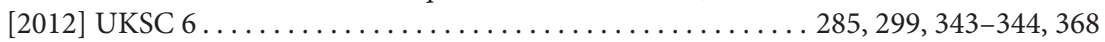

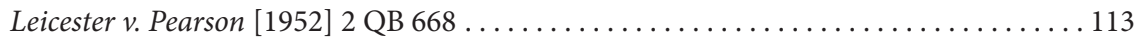
Litster v. Forth Dry Dock \& Engineering Co Ltd. [1990] 1 AC 546 (HL). . . . . . . 261, 280, 282, 289-290, 296, 298, $300,306,322,344,387$ Littlewoods Ltd. v. Revenue and Customs Commissioners [2015] EWCA Civ 515 . . . . 374 Littlewoods Retail Ltd. v. Revenue and Customs Commissioners [2010] EWHC 1071 . . . 359 Macarthys Ltd. v. Smith [1979] ICR 785 (CA) . . . . . . . . . . . . . . . . . . 363

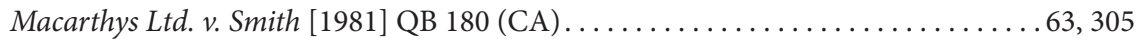
Madzimbamuto v. Lardner Burke [1969] 1 AC 645 (PC) ..................... 5 Magor and St Mellons Rural DC v. Newport Corporation

[1950] 2 All ER 1226 (CA) . . . . . . . . . . . . . . . . . . . . . . . . . . . . . 129 Magor and St Mellons Rural DC v. Newport Corporation [1952] AC 189 (HL) . . . 102-103, $106,129,175$

Manchester City Council v. Pinnock [2010] UKSC $45 \ldots \ldots \ldots \ldots \ldots \ldots$ 155, 176, 198, 315 Maunsell v. Olins [1975] AC 373 (HL).........................53, 60-62 McCartan Turkington Breen v. Times Newspapers Ltd. [2001] 2 AC 277 (HL) . ... . 52, 124 McDonald v. McDonald [2016] UKSC 28 ........................ 194, 214 McE v. Prison Service of Northern Ireland [2009] UKHL 15 . . . . . . . . . . . . . . . 248 McMonagle v. Westminster City Council [1990] 2 AC 716 (HL) ............114-115, 131 Monro v. Revenue and Customs Commissioners [2008] EWCA Civ 306............. 105 Moohan v. Lord Advocate [2014] UKSC 67.............................. 110 Moreno v. The Motor Insurers' Bureau [2016] UKSC $52 \ldots \ldots \ldots \ldots \ldots \ldots \ldots . \ldots 282,324$ Nairn v. University of St Andrews [1909] AC 147 (HL) . . . . . . . . . . . . . . . . . . 249

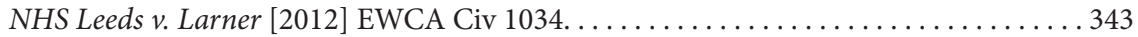
O'Brien v. Ministry of Justice [2010] UKSC $34 \ldots \ldots \ldots \ldots \ldots \ldots \ldots \ldots \ldots \ldots \ldots \ldots \ldots$ O'Brien v. Ministry of Justice [2015] EWCA Civ 1000 ...............347, 368, 374 Oakley Inc. v. Animal Ltd. [2005] EWCA Civ $1191 \ldots \ldots \ldots \ldots \ldots \ldots \ldots \ldots$. . . . . . 260, 323 Oliver Ashworth (Holdings) Ltd. v. Ballard (Kent) Ltd. [2000] Ch. 12 (CA) . . . 58, 60, 62-63 Pepper v. Hart [1993] AC 593 (HL) ..........................60, 62, 64 Perceval-Price v. Department of Economic Development [2000] IRLR 380 . . . . . . 378, 399 Percy v. Church of Scotland Board of National Mission [2005] UKHL 73 ............ 378 
Pham v. Secretary of State for the Home Department [2015] UKSC 19........... 4, 280 Pickstone v. Freemans Plc [1987] 3 WLR 811 (CA) . .......... 296, 298, 302, 323, 367 Pickstone v. Freemans Plc [1989] AC 66 (HL).................... 282, 285-286, $295-296,301,305,322-323,344-345$

Pinner v. Everett [1969] 1 WLR 1266 (HL) . . . . . . . . . . . . . . . . . . 59

Pomiechowski v. District Court of Legnica [2012] UKSC 20 . . . . . . . . . . . . . . . . 194

Poplar Housing and Regeneration Community Association Ltd.

v. Donaghue [2001] EWCA Civ 595 .................. 144, 152, 156-157, 188

Post Office v. Estuary Radio Ltd. [1968] 2 QB 740 (CA) ........................ 66

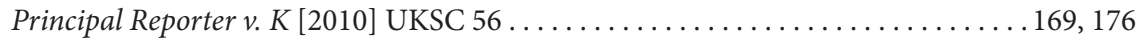

Prudential Assurance Co Ltd. v. Revenue and Customs Commissioners

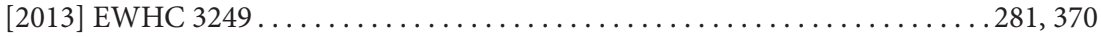

Prudential Assurance Co. Ltd. v. Revenue and Customs Commissioners

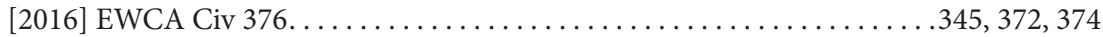

R. v. A (No. 2) [2001] UKHL $25 \ldots \ldots \ldots \ldots \ldots \ldots \ldots \ldots \ldots \ldots \ldots \ldots \ldots \ldots . \ldots \ldots, 136,152-153$,

$155-156,163,169,171-172$,

$187,190,194,198,200,203$

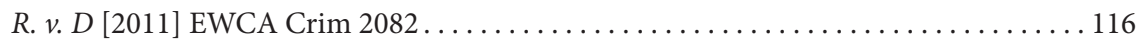

R. v. Holding [2005] EWCA Crim 3185 ................................ 169

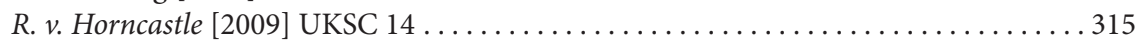

R. v. Ireland [1998] AC 147 (HL) . . . . . . . . . . . . . . . . . . . . . . 52

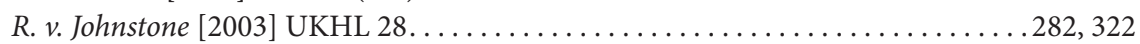

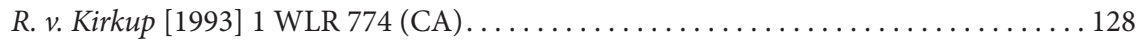

R. v. Lambert [2001] UKHL 37. .................... 147, 169, 173, 194, 200

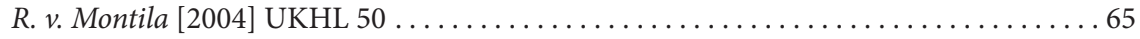

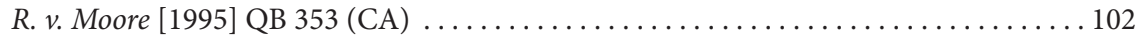

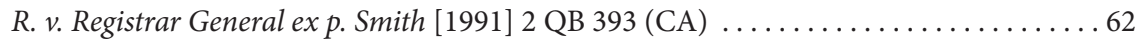

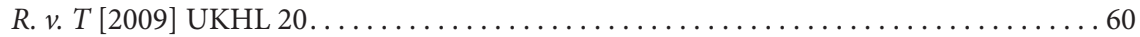

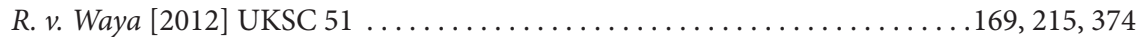

R. (Allensway Recycling Ltd) v. Environment Agency [2015] EWCA Civ 1289. . . . . . . . 118

$R$. (Anderson) v. Secretary of State for the Home Department

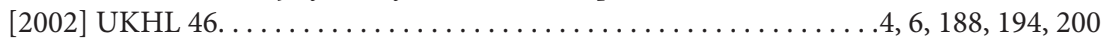

R. (Animal Defenders International) v. Secretary of State for culture,

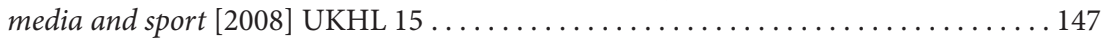

R. (Anufrijeva) v. Secretary of State for the Home Department [2003] UKHL 36 . . . 37, 250

R. (Brind) v. Secretary of State for the Home Department [1991] 1 AC 696 (HL) . . . . . . 66

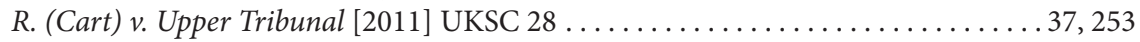

R. (Chester) v. Secretary of State for Justice [2013] UKSC 63 ................... 326

R. (Chief Constable of Staffordshire) v. Stafford Crown Court

[1999] 1 WLR 398 (QB) .................................... 113

$R$. (Confederation of Passenger Transport UK) v. Humber Bridge Board

[2003] EWCA Civ 842 . . . . . . . . . . . . . . . . . . . . . . . . . . . . . . 115

R. (Countryside Alliance) v. Attorney General [2005] EWHC 1677 (Admin) . . . . . . . . 193

R. (Doody) v. Secretary of State for the Home Department [1994] 1 AC 531 (HL). . . . . 239

R. (Edison First Power Ltd.) v. Central Valuation Officer [2003] UKHL $20 \ldots \ldots \ldots \ldots . . . .32$

R. (Equal Opportunities) v. Secretary of State for Employment [1992] ICR 341 (CA) . . . 363

R. (Evans) v. Attorney General [2015] UKSC 21 . . . . . . . . . . . . . . . . . 111, 248, 255

R. (Factortame (No. 1)) v. Secretary of State for Transport [1990] 2 AC 85 (HL) . . . 364, 367

R. (Factortame (No. 2)) v. Secretary of State for Transport

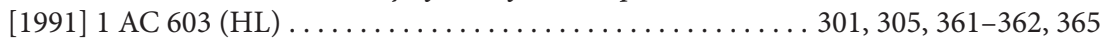


R. (Fire Brigades Union) v. Secretary of State for the Home Department [1995] 2 AC 513 (HL) .....................................

R. (Francis \& Francis) v. Central Criminal Court [1989] AC 346 (HL). . . . . . . .61, 102-103

R. (GC) v. Commissioner of Police of the Metropolis [2011] UKSC 21 ......... 163, 188, $202-203,210-215,221$

R. (Gillan) v. Commissioner of Police of the Metropolis [2006] UKHL $12 \ldots \ldots \ldots \ldots . .250$

R. (Gujra) v. Crown Prosecution Service [2012] UKSC 52 ....................... 251

R. (Hammond) v. Secretary of State for the Home Department

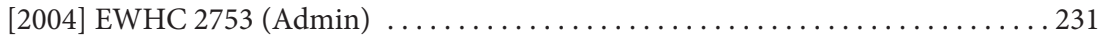

R. (Hammond) v. Secretary of State for the Home Department [2005] UKHL 69 . . . . . 148

R. (Haw) v. Secretary of State for the Home Department [2006] EWCA Civ 532....113-114

R. (HS2 Action Alliance Ltd) v. Secretary of State for Transport

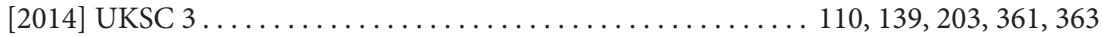

R. (Hurst) v. Commissioner of Police of the Metropolis [2007] UKHL 13 ....... 155, 190,

290, 314

R. (Irving) v. Secretary of State for Transport [2008] EWHC 1200 . . . . . . . . . . . 359

R. (Jackson) v. Attorney General [2005] UKHL 56. ..............4 37, 59, 110-112, $125,197,204-205,256,362$

R. (JS) v. Secretary of State for Work and Pensions [2015] UKSC 16 ......65, 153, 204, 280

R. (Kebilene) v. Director of Public Prosecutions [2000] 2 AC 326 (HL) ............. 146

R. (Kelly) v. Secretary of State for Justice [2008] EWCA Civ 177 . . . . . . 107, 112, 115, 118

R. (M) v. Hackney London Borough Council [2011] EWCA Civ 4.................. 169

R. (M) v. Hammersmith and Fulham London Borough Council

(1998) 30 HLR 10 (CA)....................................... 53

R. (Miller) v. Secretary of State for Exiting the European Union

[2016] EWHC 2768 (Admin) ......................... 4, 110-111, 204, $206,249,254,362-363$

R. (Miller) v. Secretary of State for Exiting the European Union

[2017] UKSC $51 \ldots \ldots \ldots \ldots \ldots \ldots \ldots \ldots \ldots \ldots . \ldots \ldots, 110-111,139,203,249,254$, $280,361-363,365-366,406$

R. (Morgan Grenfell) v. Special Commissioners of Income Tax [2002] UKHL 21 . . . 200, 249

R. (Nicklinson) v. Ministry of Justice [2013] EWCA Civ 961 . . . . . . . . . . 249-252

R. (Nicklinson) v. Ministry of Justice [2014] UKSC 38 . . . . . 11, 146, 189, 217, 220, 238

R. (Noone) v. Governor of Drake Hall Prison [2010] UKSC 30.............115, 119, 123

R. (Nutricia Ltd.) v. Secretary of State for Health [2015] EWHC 2285 . . . . . . . . 267, 300

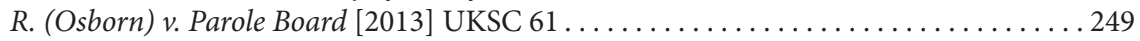

R. (Pierson) v. Secretary of State for the Home Department [1998] AC 539 (HL) ....31, 249

R. (Plantagenet Alliance Ltd) v. Secretary of State for Justice

[2014] EWHC 1662 (QB) ....................................... 109

R. (Privacy International) v. Investigatory Powers Tribunal

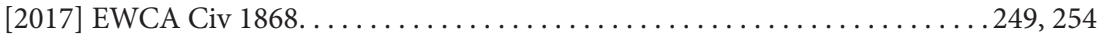

R. (Public Law Project) v. Lord Chancellor [2016] UKSC 39........... 110, 125, 305-306

R. (Quintavalle) v. Secretary of State for Health [2003] UKHL 13 . .......... 46, 51-52,

$60,62-63,107,119-122,124$

R. (Reilly) v. Secretary of State for Work and Pensions [2016] EWCA Civ 413......... 193

R. (Risk Management Partners Ltd.) v. Brent London Borough Council and Harrow London Borough Council [2011] UKSC 7 ..................... 324

R. (Roszkowski) v. Secretary of State for the Home Department

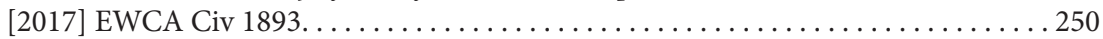

R. (Shah) v. Barnet LBC [1983] 2 AC 309 (HL) . ............................. 126 
R. (Simms) v. Secretary of State for the Home Department

[2000] 2 AC 115 (HL) ...................... 149, 163, 201, 248-250, 315

R. (Spath Holme) v. Secretary of State for the Environment, Transport

and the Regions [2001] 2 AC 349 (HL) . . . . . . . . . . . . . . . . . . 35, 37, 51-52,

$58-60,62-65,134,198$

R. (Unison) v. Lord Chancellor [2017] UKSC $51 \ldots \ldots \ldots \ldots \ldots \ldots$

R. (Westminster City Council) v. NASS [2002] UKHL $38 \ldots \ldots \ldots \ldots \ldots .663-64,67-68$

R. (Wilkinson) v. Inland Revenue Commissioners [2005] UKHL $30 \ldots \ldots \ldots \ldots .51,162$,

$193-194,198,208,230$

R. (Wright) v. Secretary of State for Health [2007] EWCA Civ 999 . . . . . . . . . . . 152, 282

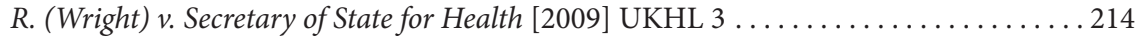

R. (ZYN) v. Walsall Metropolitan Borough Council [2014] EWHC 1918 ............. 123

Revenue and Customs Commissioners v. IDT Card Services Ireland Ltd.

[2006] EWCA Civ 29............................. 34-35, 39, 44, 261, $278,280-281,285,291,299,309$, $314,343-345,347-348,359-360$, $363-364,368,370,372-373,393$

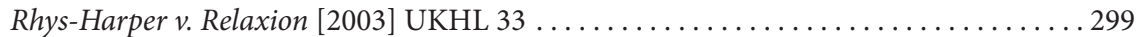
River Wear Commissioners v. Adamson (1887) 2 App. Cas. 743 ................6 62 Robertson v. Swift [2014] UKSC $50 \ldots \ldots \ldots \ldots \ldots \ldots \ldots \ldots \ldots . \ldots \ldots, 60,261,280,314$ Ropaigealach v. Barclays Bank plc. [2000] QB 263 (CA) .................... 112 Rowstock Ltd. v. Jessemey [2014] EWCA Civ 185............ 115, 118, 170, 290, 299 Royal College of Nursing of the United Kingdom v. Department

of Health and Social Security [1981] AC 800 (HL) .....................120-124

Russell v. TransOcean International Resources Ltd. [2011] UKSC 57 ............... 282

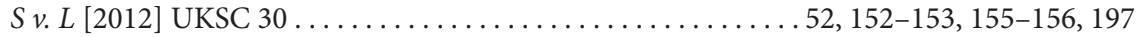
Salomon v. Commissioners of Customs and Excise [1967] 2 QB 116 (CA) . . .60, 65-66, 280 Seaford Court Estates Ltd. v. Asher [1949] 2 KB 481 (CA) ..................... 129 Secretary of State for Defence v. Guardian Newspaper Ltd. [1985] AC 339 (HL) ........ 128 Secretary of State for the Home Department v. AF [2009] UKHL 28 .... 146, 148, 194, 196 Secretary of State for the Home Department v. GG [2009] EWCA Civ 786 . . . . . . . . 248 Secretary of State for the Home Department v. JJ [2007] UKHL 45 . . . . . . . . . . . . . . 146 Secretary of State for the Home Department v. MB [2007] UKHL 46 ............... 196 Secretary of State for Work and Pensions v. Hourigan [2002] EWCA Civ 1890 . . . . . . . 62 Serious Organised Crime Agency v. Gale [2010] EWCA Civ 759 .................. 193 Shanning International Ltd. v. Lloyds TSB Bank plc [2001] UKHL $31 \ldots \ldots . . . \ldots . . .128$ Sheldon v. RHM Outhwaite (Underwriting Agencies) Ltd. [1996] AC 102 (HL) ........ 60 Simms v. Registrar of Probates [1900] AC 323 (PC) .......................... 61 Steele Ford \& Newton v. Crown Prosecution Service (No. 2) [1994] 1 AC 22 (HL) ...... 128

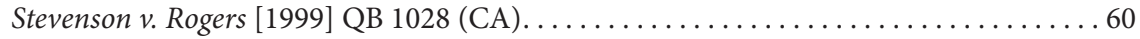
Stock v. Frank Jones (Tipton) Ltd. [1978] 1 WLR 231 (HL)................. 35, 53, $59-60,102,112,134$

Test Claimants in the FII Group Litigation v. Revenue and Customs

Commissioners [2010] EWCA Civ $103 \ldots \ldots \ldots \ldots \ldots \ldots \ldots \ldots \ldots \ldots \ldots \ldots . \ldots \ldots, 326$

Test Claimants in the FII Group Litigation v. Revenue and Customs

Commissioners [2016] EWCA Civ $1180 \ldots \ldots \ldots \ldots \ldots \ldots \ldots \ldots \ldots \ldots \ldots . \ldots \ldots . \ldots \ldots, 368$

The United States of America v. Nolan [2014] EWCA Civ $71 \ldots \ldots \ldots \ldots . \ldots . \ldots \ldots 36$

The United States of America v. Nolan [2015] UKSC 63 ......................... 374

Thoburn v. Sunderland City Council [2002] EWHC 195 (Admin) ............ 5, 110,

$139,203-205,260,363,365-366$ 
TV Broadcasting Ltd. v. TV Catchup Ltd. [2015] EWCA Civ 204 .................. 299

Unilin Beheer BV v. Berry Floor NV [2004] EWCA Civ 1021................... 282

Vacher \& Sons v. London society of compositors [1913] AC 107 (HL) . . . . . . . . . 59, 103

Victor Chandler International Ltd. v. Customs and Excise Commissioners

[2000] 1 WLR 1296 (CA) . . . . . . . . . . . . . . . . . . . . . . . . . . 124

Vodafone 2 v. Revenue and Customs Commissioners [2008] EWHC 1569 . . . . 359, 373-374

Vodafone 2 v. Revenue and Customs Commissioners [2009] EWCA Civ 446..... . 45, 189,

$201,215-216,285,299,314$,

$316,344-345,368,370-374$

Watkins v. Secretary of State for the Home Department [2006] UKHL 17 . . . . . . 139, 249

Webb v. EMO Air Cargo (UK) Ltd. (No. 2) [1995] 4 All ER 577 (HL)................. 284

Webb v. EMO Air Cargo (UK) Ltd. [1992] 2 All ER 43 (CA) ..................... 284

Webb v. EMO Air Cargo (UK) Ltd. [1992] 4 All ER 929 (HL) .................... 284

West Mercia Constabulary v. Wagener [1982] 1 WLR $127 \ldots \ldots \ldots \ldots \ldots \ldots \ldots \ldots$

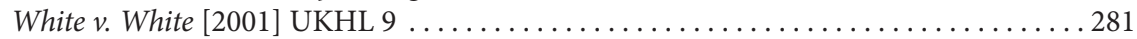

Wilson v. First County Trust Ltd. (No. 2) [2003] UKHL 40........... 51, 53, 58, 63, 65,

$67-68,145-146,152,163,192$

Yarl's Wood Immigration Ltd. v. Bedfordshire Police Authority

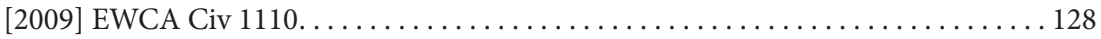

Yemshaw v. London Borough of Hounslow [2011] UKSC 3........... 52, 122, 124-125

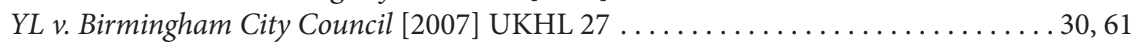




\section{LIST OF ABBREVIATIONS}

\begin{tabular}{|c|c|}
\hline AcP & Archiv für die civilistische Praxis \\
\hline AG & Advocate General \\
\hline AJCL & American Journal of Comparative Law \\
\hline All ER & All England Law Reports \\
\hline AöR & Archiv des öffentlichen Rechts \\
\hline AP & Arbeitsrechtliche Praxis \\
\hline art. & article \\
\hline BAG & Bundesarbeitsgericht (German Federal Labour Court) \\
\hline BeckRS & Beck online Rechtsprechung \\
\hline BGB & Bürgerliches Gesetzbuch (German Civil Code) \\
\hline $\mathrm{BGH}$ & Bundesgerichtshof (German Federal Court of Justice) \\
\hline BSG & Bundessozialgericht (German Federal Social Court) \\
\hline BVerfG & Bundesverfassungsgericht (German Federal Constitutional Court) \\
\hline BVerfGG & $\begin{array}{l}\text { Bundesverfassungsgerichtsgesetz (Act on the German Federal } \\
\text { Constitutional Court) }\end{array}$ \\
\hline BVerwG & Bundesverwaltungsgericht (German Federal Administrative Court) \\
\hline CA & Court of Appeal of England and Wales \\
\hline Cf. & Compare \\
\hline CJEU & Court of Justice of the European Union \\
\hline CLJ & Cambridge Law Journal \\
\hline CMLR & Common Market Law Review \\
\hline CUP & Cambridge University Press \\
\hline Deb. & Debates \\
\hline DöV & Die öffentliche Verwaltung \\
\hline DVBl & Deutsches Verwaltungsblatt \\
\hline EBLR & European Business Law Review \\
\hline $\mathrm{ECA}$ & European Communities Act 1972 \\
\hline ECHR & European Convention on Human Rights \\
\hline ECtHR & European Court of Human Rights \\
\hline EEC & (Treaty establishing the) European Economic Community \\
\hline EHRLR & European Human Rights Law Review \\
\hline EHRR & European Human Rights Reports \\
\hline EJIL & European Journal of International Law \\
\hline ELJ & European Law Journal \\
\hline ELR & European Law Review \\
\hline EU & European Union \\
\hline
\end{tabular}




\begin{tabular}{|c|c|}
\hline EuGRZ & Europäische Grundrechte-Zeitschrift \\
\hline EuR & Europarecht \\
\hline $\mathrm{EU}(\mathrm{W})$ Act & European Union (Withdrawal) Act 2018 \\
\hline EuZW & Europäische Zeitschrift für Wirtschaftsrecht \\
\hline FamRZ & Zeitschrift für das gesamte Familienrecht \\
\hline GG & Grundgesetz (German Basic Law) \\
\hline GRUR & Gewerblicher Rechtsschutz und Urheberrecht \\
\hline $\mathrm{HC}$ & House of Commons \\
\hline $\mathrm{HL}$ & House of Lords \\
\hline HRA & Human Rights Act 1998 \\
\hline ICLQ & International and Comparative Law Quarterly \\
\hline $\mathrm{I}-\mathrm{CON}$ & International Journal of Constitutional Law \\
\hline IRLR & Industrial Relations Law Reports \\
\hline JBl & Juristische Blätter \\
\hline JR & Judicial Review \\
\hline JuS & Juristische Schulung \\
\hline $\mathrm{JZ}$ & JuristenZeitung \\
\hline KG & Kammergericht (Berlin Appellate Court) \\
\hline LG & Landgericht (German Regional Court) \\
\hline LJ & Lady Justice of Appeal or Lord Justice of Appeal \\
\hline LQR & Law Quarterly Review \\
\hline MJ & Maastricht Journal of European and Comparative Law \\
\hline MLR & Modern Law Review \\
\hline MR & Master of the Rolls \\
\hline n. & note \\
\hline NJW & Neue Juristische Wochenschrift \\
\hline NVwZ & Neue Zeitschrift für Verwaltungsrecht \\
\hline NVwZ-RR & Neue Zeitschrift für Verwaltungsrecht, Rechtsprechungs-Report \\
\hline NZA & Neue Zeitschrift für Arbeitsrecht \\
\hline NZA-RR & Neue Zeitschrift für Arbeitsrecht, Rechtsprechungs-Report \\
\hline NZS & Neue Zeitschrift für Sozialrecht \\
\hline OJLS & Oxford Journal of Legal Studies \\
\hline OLG & Oberlandesgericht (German Higher Regional Court) \\
\hline OUP & Oxford University Press \\
\hline QB & Queen's Bench Division of the High Court \\
\hline PC & Privy Council of the United Kingdom \\
\hline PL & Public Law \\
\hline RabelsZ & Rabels Zeitschrift für ausländisches und internationales Privatrecht \\
\hline $\mathrm{RdA}$ & Recht der Arbeit \\
\hline RIW & Recht der internationalen Wirtschaft \\
\hline s. & section \\
\hline Sch. & Schedule \\
\hline ss. & sections \\
\hline
\end{tabular}




$\begin{array}{ll}\text { SSRN } & \text { Social Science Research Network } \\ \text { StGB } & \text { Strafgesetzbuch (German Criminal Code) } \\ \text { TEU } & \text { Treaty on European Union } \\ \text { TFEU } & \text { Treaty on the Functioning of the European Union } \\ \text { UKSC } & \text { Supreme Court of the United Kingdom } \\ \text { Vol. } & \text { Volume } \\ \text { WLR } & \text { The Weekly Law Reports } \\ \text { WM } & \text { Wertpapier-Mitteilungen, Zeitschrift für Wirtschafts- und Bankrecht } \\ \text { ZaöRV } & \text { Zeitschrift für ausländisches öffentliches Recht und Völkerrecht } \\ \text { ZEuP } & \text { Zeitschrift für Europäisches Privatrecht } \\ \text { ZfPW } & \text { Zeitschrift für die gesamte Privatrechtswissenschaft } \\ \text { ZfRV } & \text { Zeitschrift für Europarecht, Internationales Privatrecht und } \\ & \text { Rechtsvergleichung } \\ \text { ZJS } & \text { Zeitschrift für das Juristische Studium } \\ \text { ZRP } & \text { Zeitschrift für Rechtspolitik } \\ \text { ZUM } & \text { Zeitschrift für Urheber- und Medienrecht }\end{array}$


